\title{
VARIABLE VERY HIGH ENERGY $\gamma$-RAY EMISSION FROM MARKARIAN 501
}

J. Albert ${ }^{1}$ E. Aliu, ${ }^{2}$ H. Anderhub,${ }^{3}$ P. Antoranz, ${ }^{4}$ A. Armada,${ }^{2}$ C. Baixeras, ${ }^{5}$ J. A. Barrio, ${ }^{4}$ H. Bartko, ${ }^{6}$ D. Bastieri,${ }^{7}$ J. K. Becker, ${ }^{8}$ W. Bednarek, ${ }^{9}$ K. Berger, ${ }^{1}$ C. Bigongiari, ${ }^{7}$ A. Biland, ${ }^{3}$ R. K. Bock, ${ }^{6,7}$ P. Bordas, ${ }^{10}$ V. Bosch-Ramon, ${ }^{10}$ T. Bretz, ${ }^{1}$ I. Britvitch, ${ }^{3}$ M. Camara, ${ }^{4}$ E. Carmona, ${ }^{6}$ A. Chilingarian, ${ }^{11}$ J. A. Coarasa, ${ }^{6}$ S. Commichau, ${ }^{3}$ J. L. Contreras, ${ }^{4}$ J. Cortina, ${ }^{2}$ M. T. Costado, ${ }^{12,13}$ V. Curtef, ${ }^{8}$ V. Danielyan, ${ }^{11}$ F. Dazzi, ${ }^{7}$ A. De Angelis, ${ }^{14}$ C. Delgado, ${ }^{12}$ R. de los Reyes, ${ }^{4}$ B. De Lotto, ${ }^{14}$ E. Domingo-Santamaría, ${ }^{2}$ D. Dorner, ${ }^{1}$ M. Doro, ${ }^{7}$ M. Errando, ${ }^{2}$ M. Fagiolini, ${ }^{15}$ D. Ferenc, ${ }^{16}$ E. Fernández, ${ }^{2}$ R. Firpo, ${ }^{2}$ J. Flix, ${ }^{2}$ M. V. Fonseca, ${ }^{4}$ L. Font, ${ }^{5}$ M. Fuchs, ${ }^{6}$ N. Galante, ${ }^{6}$ R. J. García-López, ${ }^{12,13}$ M. GarczarczyK, ${ }^{6}$ M. Gaug, ${ }^{12}$ M. Giller, ${ }^{9}$ F. Goebel, ${ }^{6}$ D. Hakobyan, ${ }^{11}$ M. Hayashida, ${ }^{6}$ T. Hengstebeck, ${ }^{17}$ A. Herrero, ${ }^{12,13}$ D. Höhne, ${ }^{1}$ J. Hose, ${ }^{6}$ D. Hrupec,${ }^{16,18}$ C. C. Hsu, ${ }^{6}$ P. Jacon, ${ }^{9}$ T. Jogler,${ }^{6}$ R. Kosyra, ${ }^{6}$

D. Kranich, ${ }^{3}$ R. Kritzer,${ }^{1}$ A. Laille, ${ }^{16}$ E. Lindfors, ${ }^{19}$ S. Lombardi,${ }^{7}$ F. Longo, ${ }^{14}$ J. López, ${ }^{2}$ M. López, ${ }^{4}$ E. Lorenz, ${ }^{3,6}$ P. Majumdar, ${ }^{6}$ G. Maneva, ${ }^{20}$ K. Mannheim, ${ }^{1}$ O. Mansutti, ${ }^{14}$ M. Mariotti, ${ }^{7}$ M. Martínez, ${ }^{2}$ D. Mazin, ${ }^{2}$ C. Merck, ${ }^{6}$ M. Meucci, ${ }^{15}$ M. Meyer, ${ }^{1}$ J. M. Miranda ${ }^{4}$ R. Mirzoyan, ${ }^{6}$ S. Mizobuchi, ${ }^{6}$ A. Moralejo, ${ }^{2}$ D. Nieto, ${ }^{4}$ K. Nilsson, ${ }^{19}$ J. Ninkovic, ${ }^{6}$ E. Oña-Wilhelmi, ${ }^{2}$ N. Otte, ${ }^{6,17}$ I. Oya,${ }^{4}$ D. Paneque, ${ }^{6,21}$ M. Panniello, ${ }^{12,22}$ R. Paoletti, ${ }^{15}$ J. M. Paredes, ${ }^{10}$ M. Pasanen, ${ }^{19}$ D. Pascoli,${ }^{7}$ F. Pauss,${ }^{3}$ R. Pegna, ${ }^{15}$ M. Persic, ${ }^{14,23}$ L. Peruzzo, ${ }^{7}$ A. Piccioli, ${ }^{15}$ E. Prandini, ${ }^{7}$ N. Puchades, ${ }^{2}$ A. Raymers, ${ }^{11}$ W. Rhode, ${ }^{8}$ M. Ribó,${ }^{10}$ J. Rico, ${ }^{2}$ M. Rissi, ${ }^{3}$ A. Robert, ${ }^{5}$ S. Rügamer, ${ }^{1}$ A. Saggion, ${ }^{7}$ T. Saito, ${ }^{6}$ A. Sánchez, ${ }^{5}$ P. Sartori, ${ }^{7}$ V. Scalzotto, ${ }^{7}$ V. Scapin, ${ }^{14}$ R. Schmitt,${ }^{1}$

T. Schweizer, ${ }^{6}$ M. Shayduk, ${ }^{6,17}$ K. Shinozaki, ${ }^{6}$ S. N. Shore, ${ }^{24}$ N. Sidro, ${ }^{2}$ A. Sillanpää, ${ }^{19}$ D. Sobczynska, ${ }^{9}$ A. Stamerra, ${ }^{15}$ L. S. Stark, ${ }^{3}$ L. Takalo, ${ }^{19}$ F. TaVecchio, ${ }^{25}$ P. Temnikov ${ }_{15}^{20}$ D. Tescaro, ${ }^{2}$ M. Teshima, ${ }^{6}$ D. F. Torres, ${ }^{2,26}$ N. Turini, ${ }^{15}$ H. Vankov, ${ }^{20}$ V. Vitale, ${ }^{14}$ R. M. Wagner, ${ }^{6}$ T. Wibig, ${ }^{9}$

W. Wittek, ${ }^{6}$ F. Zandanel, ${ }^{7}$ R. Zanin, ${ }^{2}$ and J. Zapatero ${ }^{5}$ Received 2007 January 31; accepted 2007 June 13

\begin{abstract}
The blazar Mrk 501 was observed at energies above $0.10 \mathrm{TeV}$ with the MAGIC Telescope from 2005 May through July. The high sensitivity of the instrument enabled the determination of the flux and spectrum of the source on a nightby-night basis. Throughout our observational campaign, the flux from Mrk 501 was found to vary by an order of magnitude. Intranight flux variability with flux-doubling times down to 2 minutes was observed during the two most active nights, namely, June 30 and July 9. These are the fastest flux variations ever observed in Mrk 501. The $\sim 20$ minute long flare of July 9 showed an indication of a $4 \pm 1$ minute time delay between the peaks of $F(<0.25 \mathrm{TeV})$ and $F(>1.2 \mathrm{TeV})$, which may indicate a progressive acceleration of electrons in the emitting plasma blob. The flux variability was quantified for several energy ranges and found to increase with the energy of the $\gamma$-ray photons. The spectra hardened significantly with increasing flux, and during the two most active nights, a spectral peak was clearly detected at $0.43 \pm 0.06$ and $0.25 \pm 0.07 \mathrm{TeV}$, respectively, for June 30 and July 9 . There is no evidence of such a spectral feature for the other nights at energies down to $0.10 \mathrm{TeV}$, thus suggesting that the spectral peak is correlated with the source luminosity. These observed characteristics could be accommodated in a synchrotron self-Compton framework in which the increase in $\gamma$-ray flux is produced by a freshly injected (high energy) electron population.
\end{abstract}

Subject headings: BL Lacertae objects: individual (Mrk 501) — instrumentation: detectors (MAGIC)

Online material: color figures

1 Universität Würzburg, D-97074 Würzburg, Germany.

2 IFAE, Edifici Cn., E-08193 Bellaterra (Barcelona), Spain.

3 ETH Zurich, CH-8093 Zurich, Switzerland.

4 Universidad Complutense, E-28040 Madrid, Spain.

5 Universitat Autònoma de Barcelona, E-08193 Bellaterra, Spain.

6 Max-Planck-Institut für Physik, D-80805 Munich, Germany; dpaneque@, mppmu.mpg.de.

7 Università di Padova and INFN, I-35131 Padova, Italy.

8 Universität Dortmund, D-44227 Dortmund, Germany.

9 University of Łódź, PL-90236 Lodz, Poland.

10 Universitat de Barcelona, E-08028 Barcelona, Spain.

11 Yerevan Physics Institute, AM-375036 Yerevan, Armenia.

12 Institut de Astrofisica de Canarias, E-38200, La Laguna, Tenerife, Spain. Spain.

\footnotetext{
14 Università di Udine, and INFN Trieste, I-33100 Udine, Italy.

15 Università di Siena, and INFN Pisa, I-53100 Siena, Italy.

16 University of California, Davis, CA 95616-8677.

17 Humboldt-Universität zu Berlin, D-12489 Berlin, Germany.

18 Rudjer Boskovic Institute, Zagreb, Croatia.

19 Tuorla Observatory, Turku University, FI-21500 Piikkiö, Finland.

20 Institute for Nuclear Research and Nuclear Energy, BG-1784 Sofia, Bulgaria.

21 Corresponding author; Stanford Linear Accelerator Center, Stanford University, Menlo Park, CA 94025; dpaneque@slac.stanford.edu.

22 Deceased.

23 INAF/Osservatorio Astronomico and INFN, I-34131 Trieste, Italy.

24 Università di Pisa, and INFN Pisa, I-56126 Pisa, Italy.

25 INAF/Osservatorio Astronomico di Brera, Milan, Italy.

26 Institut de Cienciès de l'Espai, Campus UAB, E-08193 Bellaterra, Spain.
} 


\section{INTRODUCTION}

The large inferred luminosities of active galactic nuclei (AGNs) led to a standard model of beamed AGN emission, with the ultimate energy source being the release of gravitational potential energy of matter from an accretion disk surrounding a supermassive black hole (Rees 1984). Particularly interesting for the very high energy $\left(\mathrm{VHE}^{27}\right) \gamma$-ray community are the blazars, whose relativistic plasma jets point at the observer. Distinctive features of blazars are their continuum emission, clearly nonthermal from radio to VHE frequencies and characterized by two broad bumps peaking at, respectively, IR/X-ray and $\gamma$-ray frequencies (Blandford \& Rees 1978; Urry \& Padovani 1995; Ulrich et al. 1997), and their strong variability, implying flux variations by a factor of $\gtrsim 10$ over timescales of $\lesssim 1 \mathrm{hr}$ to months (flares; see Ulrich et al. 1997).

So far, 13 AGNs have been detected at VHE energies. Except for M87 (Aharonian et al. 2003, 2006b), all these sources belong to the "high-peaked BL Lac" (HBL) subclass of blazars, which are characterized by a spectral energy distribution (SED) in which both maxima occur at relatively high frequency (e.g., at hard X-rays and HE/VHE $\gamma$-rays, respectively). The detection of $\gamma$-rays from blazars leads to some important considerations about the relevant radiation processes and the physical properties of the emitting regions. Most notably, the very detection of VHE radiation, implying $\gamma$-ray transparency in the emitting region, requires the presence of relativistic beaming to decrease the intrinsic energy density of the soft target photons inside the source. The beaming reduces this value because it simultaneously decreases the intrinsic energy density of the photons and, having reduced the energy of the relevant $\gamma$-ray photons, it increases the energy of the soft target photons relevant to $e^{ \pm}$production, hence (for typical spectra) decreasing their number density (McBreen 1979; Blandford \& Königl 1979; Bassani \& Dean 1981; Mattox et al. 1993; Dondi \& Ghisellini 1995).

Two classes of emission models have been proposed to explain the TeV emission from blazars: leptonic and hadronic models. (1) In the case of the most popular leptonic models, the same population of nonthermal electrons (and possibly positrons) responsible for the radio-to-X-ray SED is also responsible for $\gamma$-ray emission, through Compton upscattering of the synchrotron photons off their own parent electrons: the synchrotron self-Compton (SSC) process (Marscher \& Gear 1985; Maraschi et al. 1992; Böttcher 2002). In other models, electrons scatter "external" photons that originate outside the jet (external Compton [EC] mod$\mathrm{els}^{28}$ ). In BL Lac objects the lack of strong emission lines suggests a minor role of ambient photons and hence supports the SSC models. (2) In hadronic models, the TeV radiation is produced by hadronic interactions of the highly relativistic baryonic outflow with the ambient medium, ${ }^{29}$ and/or by interactions of ultra-highenergy protons with synchrotron photons produced by electrons (Mannheim \& Biermann 1992), with the jet magnetic field (Aharonian 2000), or with both (Mücke et al. 2003; Atoyan \& Dermer 2003; Mannheim 1993). However, hadronic models are challenged by the blazars' observed X-ray versus VHE correlation and very rapid $\gamma$-ray variability. The SSC model is then widely believed to explain the dominant emission process in blazar jets (not

\footnotetext{
27 In this paper the VHE band is defined as the energy range $E \geq 0.1 \mathrm{TeV}$.

28 The external seed photons may, e.g., come from the accretion disk (Dermer $\&$ Schlickeiser 1993 ), or be the disk radiation scattered by the material around the disk and the jet (Sikora et al. 1994), or be radiation from the massive stars that enter the jet (Bednarek \& Protheroe 1997a), or be synchrotron radiation produced in the jet and reflected by the surrounding material (Ghisellini \& Madau 1996).

29 That is, gas and clouds drifting across the jet (Dar \& Laor 1997; Beall \& Bednarek 1999), the matter from the thick accretion disk (Bednarek 1993), or interactions inside the (dense) jet (Pohl \& Schlieckeiser 2000).
}

always in its simplest one-zone realization, as required by, e.g., "orphan flares"; see Krawczynski et al. 2004; Gliozzi et al. 2006).

In this framework, the importance of high-quality data on blazar VHE emission cannot be overestimated. In particular, valuable information can be obtained investigating (1) the rapid, possibly energy-dependent, flux variability; (2) the X-ray/optical versus VHE correlation; and (3) the X-ray and VHE spectral variability, with a potential energy shift of the synchrotron and Compton peaks. Simultaneous multiwavelength observations of such rapid variability can provide stringent tests to emission models, in particular on acceleration processes in jets. In the case of nearby $(z<0.1)$ sources, the extinction due to pair production by interaction of the blazar-emitted $\mathrm{TeV}$ photons with the (not well known) optical/IR extragalactic background light (EBL) photons is probably minor; thus, there is a smaller uncertainty in the determination of the intrinsic spectral features of the object.

In this paper we report about detailed measurements of the VHE emission of Mrk 501, demonstrating the capability of the MAGIC Telescope in the precision study of blazar physics. The BL Lac object Mrk 501 was the second established TeV blazar (Quinn et al. 1996; Bradbury et al. 1997). After a phase of moderate emission for about a year following its discovery as a TeV source, in 1997 Mrk 501 went into a state of surprisingly high activity and strong variability, becoming $>10$ times brighter (at energies $>1 \mathrm{TeV}$ ) than the Crab Nebula (Aharonian et al. 1999a, 1999b). In 1998-1999 the mean flux dropped by an order of magnitude (Aharonian et al. 2001). It is worth noticing that the HEGRA observations (with a threshold energy of $\sim 0.5 \mathrm{TeV}$ ) did not see spectral variations during the 1997 outburst, whereas it did observe in 1998-1999 a significantly softer low-state energy spectrum than in 1997. The Cerenkov Array at Themis (CAT; with a threshold energy of $0.25 \mathrm{TeV}$ ), on the other hand, did detect spectral variations during 1997 (Djanati-Ataï et al. 1999; Piron $2000,^{30}$ 2003).

The structure of the paper is as follows. In $\S 2$ we briefly describe the MAGIC data taking and analysis. In $\S \S 3$ and 4 we present and discuss the Mrk 501 VHE light curve (LC), spectrum, and their variability, during the observation campaign. In $\S 5$ we discuss the long-term LC of Mrk 501, short-term flux variability, flux spectrum correlations, and the overall SED of this object. Finally, $\S 6$ summarizes our main results.

\section{THE MAGIC TELESCOPE AND THE DATA ANALYSIS}

\subsection{The Instruments}

The observations in the VHE domain were carried out with the Major Atmospheric Gamma-ray Imaging Cerenkov (MAGIC) Telescope, located on the Canary island of La Palma (28.8 north, $17.9^{\circ}$ west) at the Roque de los Muchachos Observatory (about $2200 \mathrm{~m}$ above sea level). MAGIC started regular observations in the fall of 2004, and, with a main mirror diameter of $17 \mathrm{~m}$, it is currently the world's largest single-dish IACT. Further details about the characteristics and performance of MAGIC can be found elsewhere (Baixeras et al. 2004; Paneque 2004; Cortina et al. 2005; Gaug 2006). ${ }^{31}$

The MAGIC Collaboration also operates the optical KVA Telescope $(35 \mathrm{~cm})$. Simultaneously with the MAGIC observations, Mrk 501 was regularly observed with KVA as a part of the Tuorla Observatory blazar monitoring program. ${ }^{32}$ In this paper we also use 2-10 keV data taken with the RXTE satellite's all-sky

\footnotetext{
30 Available at http://lpnp90.in2p3.fr/ cat/Thesis/.

31 Theses for D. Paneque, M. Gaug, and T. Schweizer available at http:// wwwmagic.mppmu.mpg.de/publications/theses/.

32 See http://users.utu.fi/kani/1m/.
} 
TABLE 1

MAGIC OBSERVATION OF Mrk 501

\begin{tabular}{|c|c|c|c|c|c|c|c|c|c|}
\hline MJD Start & $\begin{array}{l}T_{\mathrm{obs}}{ }^{\mathrm{a}} \\
(\mathrm{hr})\end{array}$ & $\begin{array}{l}\mathrm{ZA}^{\mathrm{b}} \\
(\mathrm{deg})\end{array}$ & $\begin{array}{l}S_{\mathrm{comb}}{ }^{\mathrm{c}} \\
(\sigma)\end{array}$ & $\begin{array}{c}F_{>0.15 \mathrm{TeV}^{\mathrm{d}}} \\
\left(10^{-10} \text { photons cm }^{-2} \mathrm{~s}^{-1}\right)\end{array}$ & $\begin{array}{l}F_{>0.15 \mathrm{TeV}} \\
\quad(\mathrm{crab})\end{array}$ & $\begin{array}{c}K_{0}{ }^{\mathrm{e}} \\
\left(10^{-10} \text { photons } \mathrm{cm}^{-2} \mathrm{~s}^{-1}(0.3 \mathrm{TeV})^{-1}\right)\end{array}$ & $a^{\mathrm{f}}$ & $\chi^{2} / \mathrm{NDF}^{\mathrm{g}}$ & $\begin{array}{l}P^{\mathrm{h}} \\
(\%)\end{array}$ \\
\hline $53,518.980 \ldots \ldots$ & 0.75 & $19.10-28.95$ & 6.44 & $1.19 \pm 0.25$ & $0.37 \pm 0.08$ & $2.63 \pm 0.48$ & $2.17 \pm 0.25$ & $2.7 / 8$ & 95.2 \\
\hline $53,524.969 \ldots \ldots$ & 0.58 & $19.18-27.73$ & 6.98 & $2.04 \pm 0.29$ & $0.64 \pm 0.09$ & $3.71 \pm 0.53$ & $2.47 \pm 0.23$ & $1.6 / 6$ & 95.0 \\
\hline $53,526.975 \ldots \ldots$ & 0.98 & $9.96-28.94$ & 8.69 & $1.63 \pm 0.22$ & $0.51 \pm 0.07$ & $3.26 \pm 0.38$ & $2.49 \pm 0.17$ & $3.8 / 9$ & 92.4 \\
\hline $53,530.973 \ldots \ldots$ & 0.47 & $15.22-22.32$ & 6.52 & $1.53 \pm 0.32$ & $0.48 \pm 0.10$ & $2.28 \pm 0.65$ & $1.97 \pm 0.49$ & $1.1 / 3$ & 78.9 \\
\hline $53,533.933 \ldots \ldots$ & 1.63 & $12.85-30.09$ & 7.83 & $1.44 \pm 0.17$ & $0.45 \pm 0.05$ & $2.46 \pm 0.32$ & $2.55 \pm 0.19$ & $10.3 / 8$ & 24.2 \\
\hline $53,534.940 \ldots \ldots$ & 2.07 & $9.95-30.09$ & 9.56 & $1.43 \pm 0.15$ & $0.45 \pm 0.05$ & $2.71 \pm 0.27$ & $2.68 \pm 0.16$ & $8.9 / 9$ & 44.8 \\
\hline $53,535.934 \ldots \ldots$ & 3.43 & $9.95-30.07$ & 18.58 & $2.69 \pm 0.13$ & $0.85 \pm 0.04$ & $4.45 \pm 0.24$ & $2.42 \pm 0.06$ & $11.9 / 12$ & 45.3 \\
\hline $53,536.947 \ldots \ldots$ & 2.68 & $9.95-29.93$ & 7.01 & $0.75 \pm 0.13$ & $0.24 \pm 0.04$ & $1.36 \pm 0.21$ & $2.73 \pm 0.29$ & $5.7 / 7$ & 57.1 \\
\hline $53,537.971 \ldots \ldots$ & 3.08 & $9.95-30.10$ & 11.52 & $1.25 \pm 0.10$ & $0.39 \pm 0.03$ & $2.08 \pm 0.19$ & $2.46 \pm 0.14$ & $8.2 / 8$ & 41.4 \\
\hline $53,548.931 \ldots \ldots$ & 0.87 & $9.98-20.68$ & 6.12 & $1.21 \pm 0.25$ & $0.38 \pm 0.08$ & $2.39 \pm 0.38$ & $2.28 \pm 0.27$ & $0.6 / 6$ & 99.6 \\
\hline $53,551.905 \ldots \ldots$ & 1.09 & $12.86-25.15$ & 32.02 & $11.08 \pm 0.32$ & $3.48 \pm 0.10$ & $17.37 \pm 0.51$ & $2.09 \pm 0.03$ & $26.2 / 11$ & 0.6 \\
\hline $53,560.906 \ldots \ldots$ & 0.76 & $9.96-19.07$ & 24.39 & $9.93 \pm 0.38$ & $3.12 \pm 0.12$ & $14.35 \pm 0.56$ & $2.20 \pm 0.04$ & $22.5 / 11$ & 2.1 \\
\hline $53,562.911 \ldots \ldots$ & 1.63 & $9.94-16.79$ & 11.08 & $2.19 \pm 0.37$ & $0.69 \pm 0.12$ & $2.83 \pm 0.30$ & $2.34 \pm 0.13$ & $14.1 / 8$ & 8.2 \\
\hline $53,563.921 \ldots \ldots$ & 0.85 & $9.94-15.16$ & 18.69 & $5.53 \pm 0.28$ & $1.74 \pm 0.09$ & $7.89 \pm 0.39$ & $2.25 \pm 0.06$ & $11.5 / 9$ & 24.3 \\
\hline $53,564.917 \ldots \ldots$ & 0.34 & $9.94-15.18$ & 8.91 & $2.89 \pm 0.46$ & $0.91 \pm 0.15$ & $4.88 \pm 0.56$ & $2.27 \pm 0.20$ & $5.4 / 6$ & 49.7 \\
\hline $53,565.920 \ldots \ldots$ & 2.57 & $9.95-28.93$ & 11.62 & $1.71 \pm 0.13$ & $0.54 \pm 0.04$ & $2.73 \pm 0.22$ & $2.49 \pm 0.12$ & $10.7 / 8$ & 21.6 \\
\hline $53,566.953 \ldots \ldots$ & 1.91 & $9.99-30.10$ & 11.63 & $1.33 \pm 0.11$ & $0.42 \pm 0.04$ & $2.16 \pm 0.20$ & $2.28 \pm 0.13$ & $7.4 / 10$ & 69.0 \\
\hline
\end{tabular}

${ }^{a}$ Net observation time after removing bad-quality runs.

${ }^{b}$ Zenith angle range covered during the observation.

c Combined significance of detected signal in the $0.1-10 \mathrm{TeV}$ band.

${ }^{\mathrm{d}}$ Integrated flux above $0.15 \mathrm{TeV}$.

e Normalization factor of the PL fit.

f Slope of PL fit.

g The $\chi^{2}$ value and number of degrees of freedom of the PL fit.

${ }^{\mathrm{h}}$ Chance probability for larger $\chi^{2}$ values.

monitor (RXTE ASM $)^{33}$ quasi-simultaneously with our MAGIC observations.

\subsection{Source Observation}

The source was observed during 30 nights between 2005 May and July, with an overall observation time of $54.8 \mathrm{hr}$. In order to maximize the time coverage of this source, observations were carried out also in the presence of moonlight $(34.1 \mathrm{hr}$, i.e., $62 \%$ of the total observing time). It is important to note that many of these "Moon observations" were performed when the Moon was only partly illuminated, and mostly located at a large angle $\left(60^{\circ}-90^{\circ}\right)$ with respect to the position of Mrk 501. This kept the night-sky background (NSB) of these observations rather low and comparable to that of the moonless observations. The observations were mostly performed in the so-called on mode in which the telescope points exactly to the source (on-data), and thus its (optical) image is right in the center of the camera. The telescope was also operated in "off mode," in which it points to regions of the sky where there are no known $\gamma$-ray sources (off-data). These observations were carried out at comparable zenith angle (ZA) and NSB conditions and can therefore be used to estimate the background content of the on-data. The off-data observation time amounts to $3.5 \mathrm{hr}$.

The data were screened for hardware problems, non-optimal weather conditions, and too high NSB light. In addition, the very few runs with ZA larger than $30^{\circ}$ that survived those filters were

33 The data are publicly available at http:// heasarc.gsfc.nasa.gov/xte_weather/. also removed, in order to have a more uniform data set. The number of nights surviving these selection cuts is 24 , with a total net observation time of $31.6 \mathrm{hr} .{ }^{34}$ These observation nights, together with the corresponding net times and ZA ranges of the data acquisition, are listed in Table 1.

\subsection{Data Analysis}

The analysis used in this paper is based on the Hillas image parameters ALPHA, WIDTH, LENGTH, DIST, and SIZE to quantify the camera image (see Hillas 1985). These parameters are calculated using the calibrated signals from the individual pixels of the camera. The procedures used to calibrate the photomultiplier tube (PMT) signals in MAGIC are described in detail in Gaug (2006) and Albert (2006): here we used the Sliding Window signal extractor and performed the calibration with the excess noise factor method. Only PMT signals with more than 10 photoelectrons (eight photoelectrons for pixels in the boundary of an image) that occur within a time window of $6.6 \mathrm{~ns}$ (two FADC slices) of the neighboring pixel signal were used. The minimum total image light content (SIZE) considered in this analysis is 150 photoelectrons. The signal/background separation is achieved by applying dynamical cuts (defined as second-order polynomial functions of $\log$ SIZE) on the parameters WIDTH, LENGTH (shape parameters), and DIST (position of the image). The background contained in the on-data after the $\gamma /$ hadron separation cuts is estimated

\footnotetext{
34 Five out of the six entirely rejected observation nights (with a total observation time of $9.6 \mathrm{hr}$ ) were moonlight observations.
} 
by means of a second-order polynomial fit (with no linear term) to the ALPHA distribution from the normalized (according to the onoff observation times) off-data.

The energy of the incoming $\gamma$-rays is essentially proportional to the light content of the image (SIZE), with corrections according to the values of LENGTH, DIST, and LEAKAGE. ${ }^{35}$ The energy resolution achieved with this parameterization is about $20 \%-30 \%$, slightly depending on the event's energy. Because of the finite experimental resolution, the distribution of the excess events ( $\gamma$-candidates) versus the reconstructed energy is a convolution of the (true) energy distribution of excess events and a realistic energy resolution function. The determination of the true energy spectrum from the reconstructed one is achieved by means of an unfolding procedure (Anykeyev et al. 1991). We used the iterative method described in Bertero (1989).

In this analysis, $90 \%-99 \%$ of the background images are removed by the selection cuts, while $50 \%-60 \%$ of the $\gamma$-ray signals are kept. The resulting collection area after analysis cuts is $\gtrsim 0.5 \times 10^{5} \mathrm{~m}^{2}$ down to $0.20 \mathrm{TeV}$. The analysis threshold energy, commonly defined as the peak of the differential event rate spectrum after all cuts, is $\sim 0.15 \mathrm{TeV}$. The lowest $\gamma$-ray energy used in the calculation of the energy spectra is $0.10 \mathrm{TeV}$. In the LC analysis, however, the minimum energy considered is $0.15 \mathrm{TeV}$ (i.e., the threshold energy). Below this energy the collection area drops fast, increasing rapidly both systematic and statistical errors in the measured flux. Keeping the measurement errors small is essential for the study of flux variations, one of the main goals of this work.

In order to check the reliability of the used analysis chain, we analyzed data from the Crab Nebula taken in 2005 December, under similar instrumental and environmental conditions to those of Mrk 501. The obtained results were in perfect agreement with data published previously (Hillas et al. 1998; Aharonian et al. 2004; Wagner et al. 2005), which shows that the analysis procedures used produce reliable results.

The results from the MAGIC Mrk 501 data analysis are summarized in Table 1. The table shows the integrated flux (above $0.15 \mathrm{TeV}$ ) and the resulting fit to the differential (energy) photon spectra with a simple power-law (PL) model (see eq. [5]), for each observing night. The fit was obtained using all the spectral points above $0.10 \mathrm{TeV}$. Only statistical errors are reported in Table 1 . The systematic errors on the energy determination are estimated as $\sim 20 \%$, which, for a spectral index of 2.5 , would produce a systematic shift of $50 \%$ in the flux level (normalization factor of the PL function from Table 1). The systematic error in the calculated spectral indices is evaluated as $\sim 0.1$. In the table we quote the combined significance, which is calculated following the prescription given by Bityukov et al. (2006) as

$$
S_{\mathrm{comb}}=\frac{\sum S_{i}}{\sqrt{n}},
$$

where $S_{i}$ is the significance corresponding to the (differential) energy bin $i$ and $n$ is the number of energy bins (measurements). The significance of each energy bin is calculated according to equation (5) of Li \& Ma (1983), which is more suitable than equation (17) from the same paper, since in all nights the $\gamma$-ray signal is clear and hence its existence is not in doubt. The combined

\footnotetext{
35 LEAKAGE is defined as the fraction of the light content recorded by the outer ring of the PMT camera, and it is typically used to evaluate the level of missing light in the detected image.
}

significance is used to compare the quality of the $\gamma$-ray signals from different observing nights.

\section{LIGHT CURVE OF Mrk 501 DURING THE MAGIC OBSERVATIONS}

In this section we report on the broadband (optical to $\gamma$-ray) LC of Mrk 501 during 2005 May-July.

\subsection{Overall Light Curve at $\gamma-$ Ray, X-Ray, and Optical Frequencies}

The overall LC of Mrk 501 during the MAGIC observation campaign is shown in Figure 1. The observed flux is shown in three energy bands: VHE $(0.15-10 \mathrm{TeV}), \mathrm{X}$-rays $(2-10 \mathrm{keV})$, and optical (1.5-2.5 eV) as measured by MAGIC, RXTE ASM, and KVA, respectively. The X-ray and optical fluxes are computed as weighted averages using RXTE ASM and KVA measurements taken simultaneously with the MAGIC observations plus/minus a time tolerance of 0.2 days. A smaller time tolerance substantially decreases the number of X-ray points that can be used. The flux level of the Crab Nebula (top panel, dashed horizontal line) is also shown in Figure 1 for comparison. The Crab Nebula flux was obtained by applying the very same analysis as described in $\S 2.3$ to the MAGIC Crab Nebula data taken during 2005 December under observing conditions similar to those for Mrk 501. The estimated Crab Nebula flux level is therefore roughly affected by the same systematics as the fluxes obtained for Mrk 501 . We found $F_{\text {Crab }}(>0.15 \mathrm{TeV})=(3.2 \pm 0.1) \times 10^{-10} \mathrm{~cm}^{-2} \mathrm{~s}^{-1}$, hereafter referred to as crab units (crab). For simplicity, only the Crab Nebula flux level, and not the associated error (which is irrelevant for the comparison), is shown in the LC.

The measured VHE flux from Mrk 501 was at about $0.5 \mathrm{crab}$ during most of the observation nights (Table 1). During several nights, however, its flux significantly exceeded $0.5 \mathrm{crab}$, and during one night (MJD 53,536.947) it showed a substantially lower flux $(0.24 \pm 0.04 \mathrm{crab})$. Often, Mrk 501 showed large flux variations in consecutive nights. An example of these rapid flux variations are the MJD 53,535.934 and 53,536.947 with respective fluxes of $0.84 \pm 0.04$ and $0.24 \pm 0.04 \mathrm{crab}$, the MJD $53,554.906$ and 53,555.914 with respective fluxes of $1.11 \pm 0.09$ and $0.40 \pm 0.11 \mathrm{crab}$, and the MJD 53,563.921 and 53,564.917 with respective fluxes of $1.74 \pm 0.09$ and $0.91 \pm 0.15 \mathrm{crab}$. Besides, the VHE flux from Mrk 501 was outstanding during the MJD $53,551.905$ (June 30) and 53,560.906 (July 09) with $3.48 \pm 0.10$ and $3.12 \pm 0.12 \mathrm{crab}$, respectively. During these two nights the source was in a very active state. Note, however, that the night before the July 9 flare the emitted flux was $0.58 \pm 0.07 \mathrm{crab}$, i.e., close to the average flux of the entire campaign. Mrk 501 therefore showed a remarkably fast VHE variability during this campaign.

Unlike in VHE $\gamma$-rays, no significant flux variation was recorded in the X-ray and optical bands. In the case of the X-ray data, however, although the sensitivity of the RXTE ASM instrument was clearly inadequate to reveal short-term 2-10 keV flux variability in Mrk 501's emission, the flux appears to be higher in the second portion of the LC. The optical flux, on the other hand, shows only a modest variation, a $\sim 5 \%$ monotonic decrease during the entire observational campaign.

\subsection{Multifrequency Correlations}

The correlations of our observed VHE $\gamma$-ray data with X-ray and optical data are shown in Figure 2, the gamma points being the same as shown in the LC of Figure 1. It can be seen that the measurement uncertainties of the X-ray and optical fluxes are 

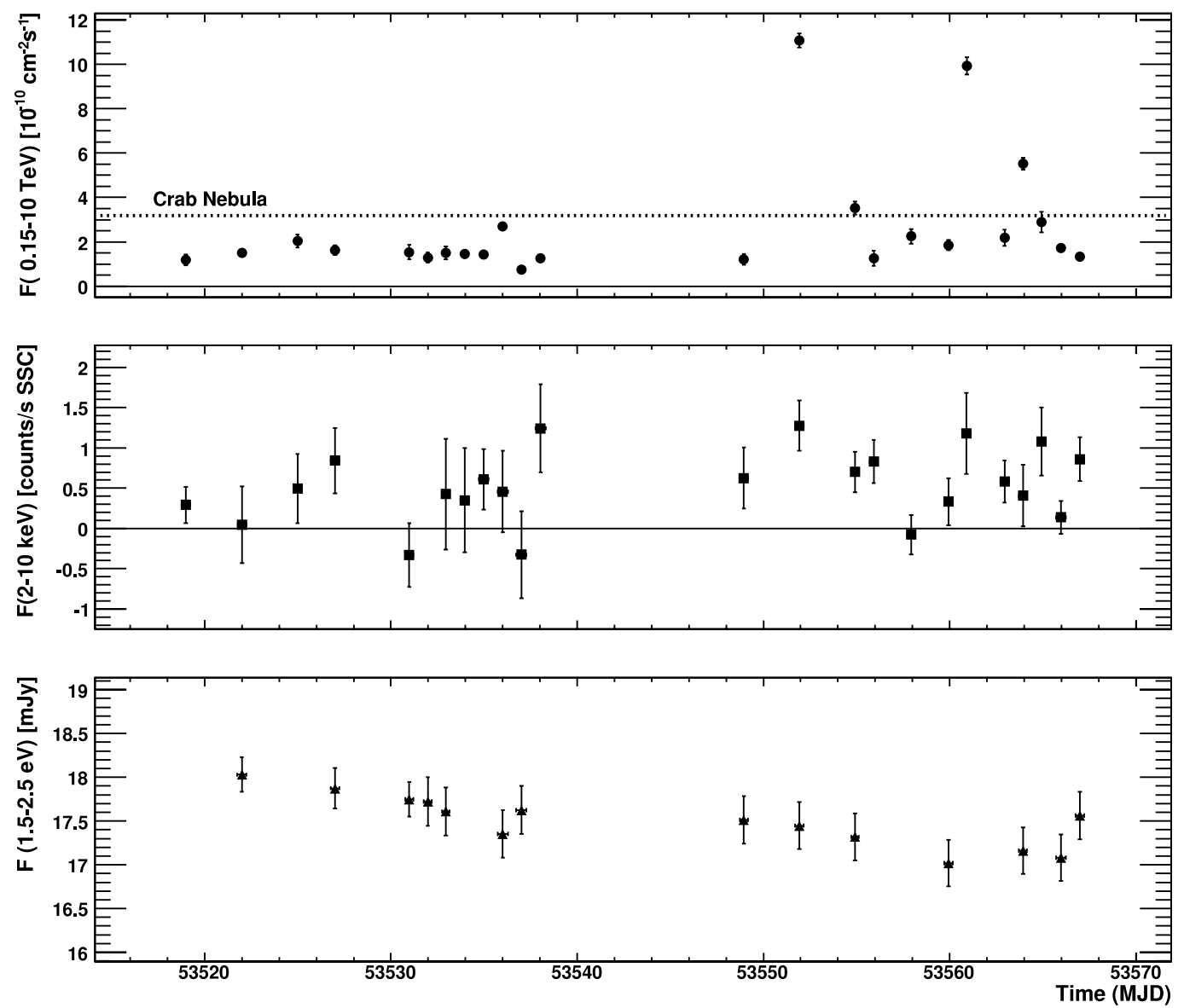

Fig. 1.-Multifrequency LC during the MAGIC observations of Mrk 501 (2005 May-July). Top: MAGIC flux above 0.15 TeV. The Crab flux is also shown for comparison (dotted horizontal line). Middle: RXTE ASM 2-10 keV flux. Bottom: KVA $\sim 1.5-2.5 \mathrm{eV}$ flux. Error bars denote $1 \sigma$ statistical uncertainties. The X-ray/optical data were selected to match the MAGIC data within a time window of 0.2 days. [See the electronic edition of the Journal for a color version of this figure.]
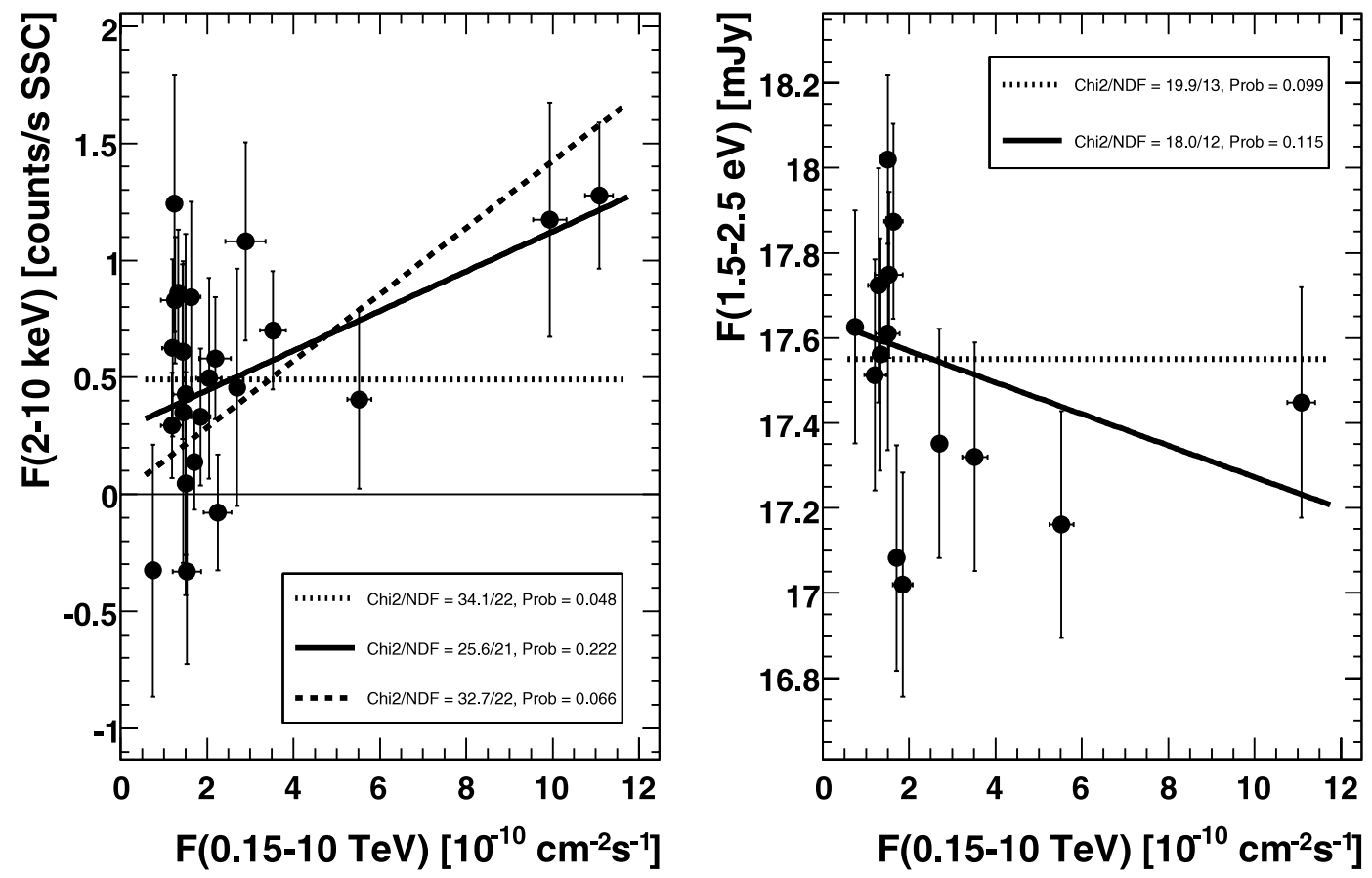

FIG. 2.-VHE vs. X-ray (left) and optical (right) flux correlation during the MAGIC observation campaign. The data points are the same as in Fig. 1. The dotted lines denote constant fits; solid and dashed lines correspond to linear fits with/without offset (see insets for goodness-of-fits values). [See the electronic edition of the Journal for a color version of this figure.] 


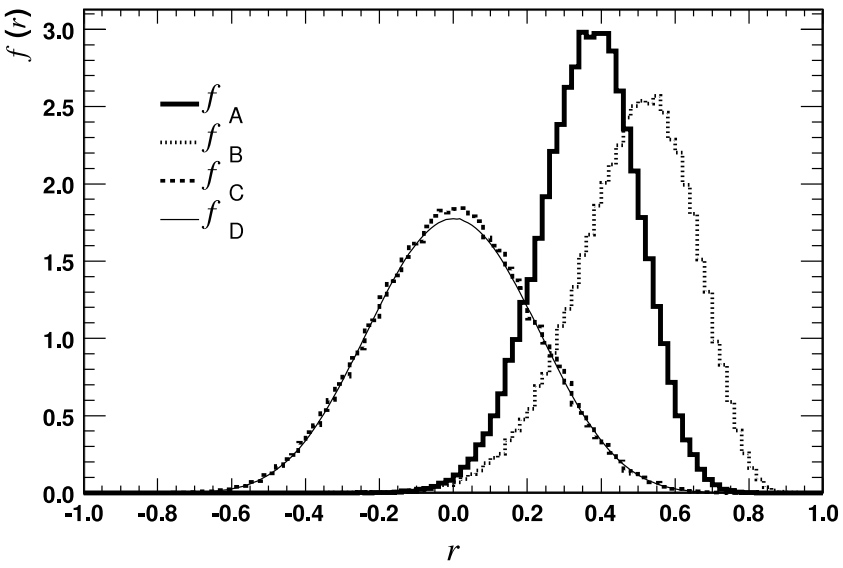

FIG. 3.-The pdf's of the correlation coefficient between $\gamma$-ray and X-ray fluxes: $f_{\mathrm{A}}$ data, $f_{\mathrm{B}}$ perfectly correlated case, $f_{\mathrm{C}}$ uncorrelated case, and $f_{\mathrm{D}}$ analytical solution in the uncorrelated case. See text for further details. [See the electronic edition of the Journal for a color version of this figure.]

comparatively large, which makes possible different dependencies of the X-ray flux on the $\gamma$-ray flux. We fit the VHE/X-ray data with a constant and with a linear function, obtaining the highest probability for the linear function. For the relation VHE/optical data, the two fits are nearly equally probable. All fit results are shown in the insets of Figure 2, including for the VHE/X-ray data also a fit forcing the linear function through the origin.

For the VHE/X-ray correlation, one obtains a linear correlation coefficient of 0.49 . Investigating the uncertainty of this result, we used a procedure of Monte Carlo-generated correlations, as described in detail by D. Ferenc \& D. Hrupec (2008, in preparation) and Hrupec (2007). For all points (corresponding VHE and X-ray fluxes), multiple (we used 100,000) possible sets of measurements are generated, using random differences derived from the (Gaussian) errors assigned to each point. For each set of generated points, a correlation coefficient is obtained, resulting in a large number of measurements in the probability density function (pdf) of correlation coefficients $f_{A}(r)$ shown in Figure 3, which corresponds to the measured correlation and the assigned errors. This pdf correctly expresses the effect of the measurement uncertainties. The same procedure can be applied to hypothetical fully correlated data: to this end, the data points are shifted onto the straight line from the fit with the highest probability (black line in left panel of Fig. 2), maintaining the original error assignments. In the case of hypothetical uncorrelated data, the points are randomly distributed in the VHE/X-ray plane. Sets of Monte Carlo measurements are then generated as before. The resulting pdf's express the probability to obtain certain values of correlation coefficients, given, respectively, no correlation $\left[f_{C}(r)\right.$ in Fig. 3] or full correlation $\left[f_{B}(r)\right.$ in Fig. 3] with our error assumptions. For comparison purposes, Figure 3 also shows the analytical pdf for the uncorrelated case, $f_{D}(r)$ (described in pp. 220 and 290 of Taylor 1997), which does not take into account measurement errors. Note that $f_{C}(r)$ is very similar to $f_{D}(r)$. This is not surprising, since the smearing does not increase the randomness of the already randomized seed event. The width of the probability density distribution $f_{D}(r)$ depends exclusively on the number of points in the data sample ( 23 in our case). On the other hand, $f_{B}(r)$ is significantly affected by the measurement errors; even under the hypothesis of a fully correlated case, the probability to obtain values for the correlation coefficient larger than 0.8 is very small.

A measure of the probability of correlation can be derived from the comparison of the probability density distribution $f_{A}(r)$

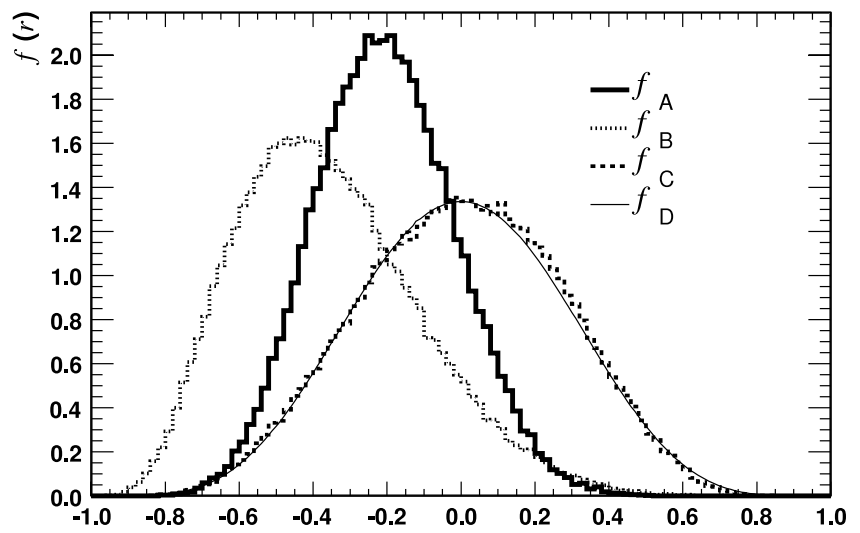

FIG. 4.-The pdf's of the correlation coefficient between $\gamma$-ray and optical fluxes: $f_{\mathrm{A}}$ data, $f_{\mathrm{B}}$ perfectly correlated case, $f_{\mathrm{C}}$ uncorrelated case, and $f_{\mathrm{D}}$ analytical solution in the uncorrelated case. See text for further details. [See the electronic edition of the Journal for a color version of this figure.]

for the actual measurement with the distributions for the two extreme correlation cases, $f_{B}(r)$ and $f_{C}(r)$. Given the results in Figure 3 , it is evident that $f_{A}(r)$ is similar to $f_{B}(r)$ but, despite a sizable overlap, rather different from $f_{C}(r)$. For a quantitative comparison, we followed the robust method from Poe et al. (2005), which is based on the convolution of empirical probability density distributions. The comparison of a pair of pdf's $f_{X}(r)$ and $f_{Y}(r)$ leads to the probability that these two distributions are statistically consistent, $P\left(f_{X}(r), f_{Y}(r)\right)$. The resulting value for the probability of agreement between our data points and the fully correlated case, $P_{X, \gamma}\left(f_{A}, f_{B}\right)$, is $0.55 \pm 0.15$. The quoted error is a systematic estimated through variations in the initial values used in the Monte Carlo method (D. Ferenc \& D. Hrupec 2008, in preparation; Hrupec 2007). The probability that our measurement came as a result of a statistical fluctuation of an entirely uncorrelated physics case, on the other hand, is significantly lower; $P_{X, \gamma}\left(f_{A}, f_{C}\right)$ is $0.15 \pm 0.05$. The probability for the first scenario to be true and for the second to be false is $P_{X, \gamma}\left(f_{A}, f_{B}\right)\left[1-P_{X, \gamma}\left(f_{A}, f_{C}\right)\right]=0.47$, and the probability for the opposite is $\left[1-P_{X, \gamma}\left(f_{A}, f_{B}\right)\right] P_{X, \gamma}\left(f_{A}, f_{C}\right)=0.067$, which indicates that the correlation scenario is significantly more likely. ${ }^{36}$

The same method was applied to the optical and $\gamma$-ray flux values of Figure 2 (right panel). The corresponding probability density functions are presented in Figure 4 . In this case, the resulting linear correlation coefficient is -0.27 , indicating a small anticorrelation; yet the probabilities $P_{\text {opt }, \gamma}\left(f_{A}, f_{B}\right)=0.60 \pm 0.25$ and $P_{\mathrm{opt}, \gamma}\left(f_{A}, f_{C}\right)=0.55 \pm 0.25$ are practically equal, which suggests that neither the anticorrelated nor the uncorrelated scenario may be reliably excluded. The probability for the first scenario to be true and for the second to be false is 0.27 , while the probability for the opposite case is 0.22 , which confirms the previous conclusion.

\subsection{Intraday $\gamma$-Ray Flux Variations}

During the two nights with the highest VHE activity, namely, June 30 and July 9, Mrk 501 clearly showed intranight flux variations. The corresponding $\mathrm{LC}$ in the $0.15-10 \mathrm{TeV}$ band is shown in Figure 5 with a time binning of $\sim 2$ minutes. For comparison, the Crab Nebula flux is shown as a dashed horizontal line. The vertical dot-dashed line divides the data into a region of relatively "stable" (preburst) emission and one of "variable" (in-burst)

36 It is worth noticing that the present analysis treats data from low and very high activity epochs together, whereas the same correlation slope may not be necessarily the same for the two activity states. 

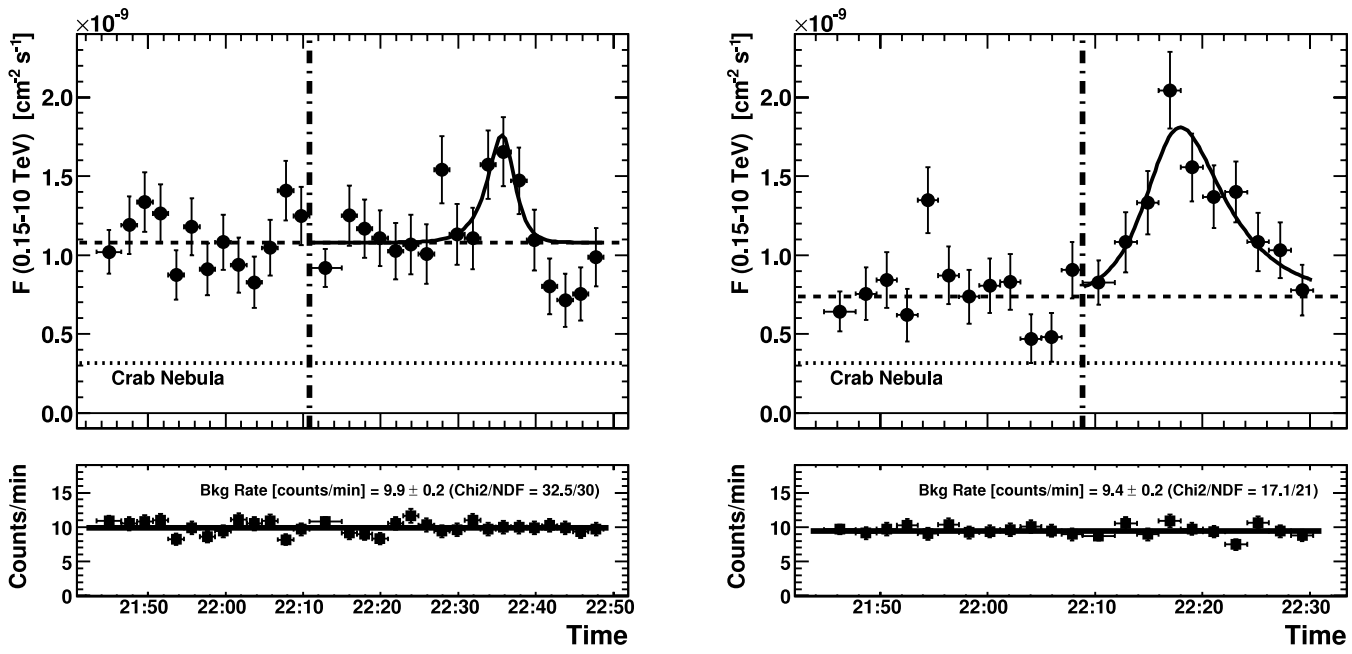

FIG. 5.- Integrated flux LCs of Mrk 501 for the flare nights of June 30 and July 9. Horizontal bars represent the 2 minute time bins, and vertical bars denote $1 \sigma$ statistical uncertainties. For comparison, the Crab emission is also shown as a dotted horizontal line. The vertical dot-dashed line divides the data into stable (i.e., preburst) and variable (i.e., in-burst) emission. The horizontal dashed line represents the average of the stable emission. The solid line represents the best-fit flare model (see eq. [2]). The bottom panels show the mean background rate during each of the 2 minute bins of the LCs. The insets report the mean background rate during the entire night, resulting from a constant fit to the data points. The goodness of such a fit is also given. [See the electronic edition of the Journal for a color version of this figure.]

emission. The background rate after the gamma/hadron selection cuts was evaluated during these two nights and is shown in the bottom panels of Figure 5. These rates were found to be constant along the entire night. Consequently, the variations seen in the top panels of Figure 5 correspond to actual variations of the VHE $\gamma$-ray flux from Mrk 501, thus ruling out detector instabilities or atmospheric changes.

A constant line fit to the whole LC gives a $\chi^{2} / \mathrm{NDF}=47.9 / 30$ (probability $P=2.0 \times 10^{-2}$ ) for the night of June 30 and a $\chi^{2} / \mathrm{NDF}=80.6 / 21\left(P=6.4 \times 10^{-9}\right)$ for the night of July 9 . The emission above $0.15 \mathrm{TeV}$ during the two nights is therefore statistically inconsistent with being constant and it is more reasonable to only fit the first part, i.e., the stable emission, with a constant, and not the entire LC. A constant fit to the stable portion of the LCs gives $\chi^{2} / \mathrm{NDF}=13.4 / 12(P=0.34)$ for June 30 and $\chi^{2} / \mathrm{NDF}=$ $17.8 / 11(P=0.09)$ for July 9 (see Fig. 5$)$. The probability that the variable parts of the LCs are compatible with the stable flux level is given by $\chi^{2} / \mathrm{NDF}=34.5 / 18\left(P=1.1 \times 10^{-2}\right)$ for June 30 and $\chi^{2} / \mathrm{NDF}=83.3 / 10\left(P=1.1 \times 10^{-13}\right)$ for July 9 . We therefore measured intranight flux variations in both nights.

The flare's amplitude and duration, as well as its rise/fall times, can be quantified according to

$$
F(t)=a+\frac{b}{2^{-\left(t-t_{0}\right) / c}+2^{\left(t-t_{0}\right) / d}}
$$

(Schweizer 2004; see footnote 31). This model parameterizes a flux variation (flare) superposed on a stable emission: $F(t)$ asymptotically tends to $a$ when $t \rightarrow \pm \infty$. The parameter $a$ is the assumed constant flux at the time of the flare (see the horizontal black dashed lines in Fig. 5); $t_{0}$ is set to the time corresponding to the point with the highest value in the LC; and $b, c$, and $d$ are left free to vary. The latter two parameters denote the flux-doubling rise and fall times, respectively, and can be converted into the characteristic rise/fall times ${ }^{37}$ by multiplying them by $1 / \ln 2$. The resulting fits using equation (2) are shown in Figure 5, and their parameter values are reported in Table 2 . In both cases, the measured rise/fall flux-doubling times are $\sim 2$ minutes, which yields characteristic rise/fall times of $2 / \ln 2 \sim 3$ minutes. These are the shortest flux variation timescales ever measured from Mrk 501, at any wavelength.

Because of the steeply falling spectra, the low-energy events dominate the LCs shown in Figure 5 and tend to hide any higher energy features. We therefore split the data into four distinct energy ranges: $0.15-0.25 \mathrm{TeV}, 0.25-0.6 \mathrm{TeV}, 0.6-1.2 \mathrm{TeV}$, and $1.2-10 \mathrm{TeV}$. The corresponding LCs for the night June 30 are shown in Figure 6. Due to the reduced photon statistics, we increased the time binning from 2 to 4 minutes. We found that only

\footnotetext{
37 The characteristic time is defined as the time needed for the flux to change by $e^{ \pm 1}$.
}

TABLE 2

Flare Model Parameters: Integral Emission above $0.15 \mathrm{TeV}$

\begin{tabular}{|c|c|c|c|c|c|c|c|c|c|}
\hline Date & $\begin{array}{l}T_{\mathrm{obs}}{ }^{\mathrm{a}} \\
(\mathrm{hr})\end{array}$ & $\begin{array}{l}S_{\text {comb }}{ }^{\mathrm{b}} \\
(\sigma)\end{array}$ & $\begin{array}{c}a^{\mathrm{c}} \\
\left(10^{-10} \text { photons } \mathrm{cm}^{-2} \mathrm{~s}^{-1}\right)\end{array}$ & $\begin{array}{c}a \\
\text { (crab) }\end{array}$ & $\begin{array}{c}b \\
\left(10^{-10} \text { photons } \mathrm{cm}^{-2} \mathrm{~s}^{-1}\right)\end{array}$ & $\begin{array}{l}c \\
(\mathrm{~s})\end{array}$ & $\begin{array}{l}d \\
(\mathrm{~s})\end{array}$ & $\chi^{2} / \mathrm{NDF}^{\mathrm{d}}$ & $\begin{array}{l}P^{\mathrm{e}} \\
(\%)\end{array}$ \\
\hline Jun $30 \ldots \ldots \ldots \ldots \ldots \ldots$ & 0.63 & 24.7 & $10.80 \pm 0.48$ & $3.39 \pm 0.15$ & $13.2 \pm 4.7$ & $81 \pm 41$ & $50 \pm 23$ & $20.0 / 15$ & $17.3^{\mathrm{f}}$ \\
\hline
\end{tabular}

\footnotetext{
a Net observation time during variable emission (right part of the graphs).

${ }^{b}$ Combined signal significance from variable emission (right part of the graphs) in $0.1-10 \mathrm{TeV}$ band.

c Integrated flux above $0.15 \mathrm{TeV}$ for the steady emission (left part of the graphs).

d The $\chi^{2}$ value and number of degrees of freedom of the fit with eq. (2).

e Chance probability of having larger $\chi^{2}$ values.

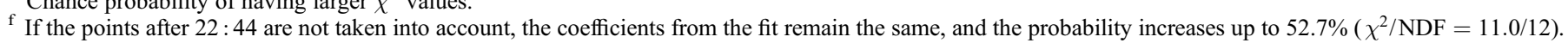



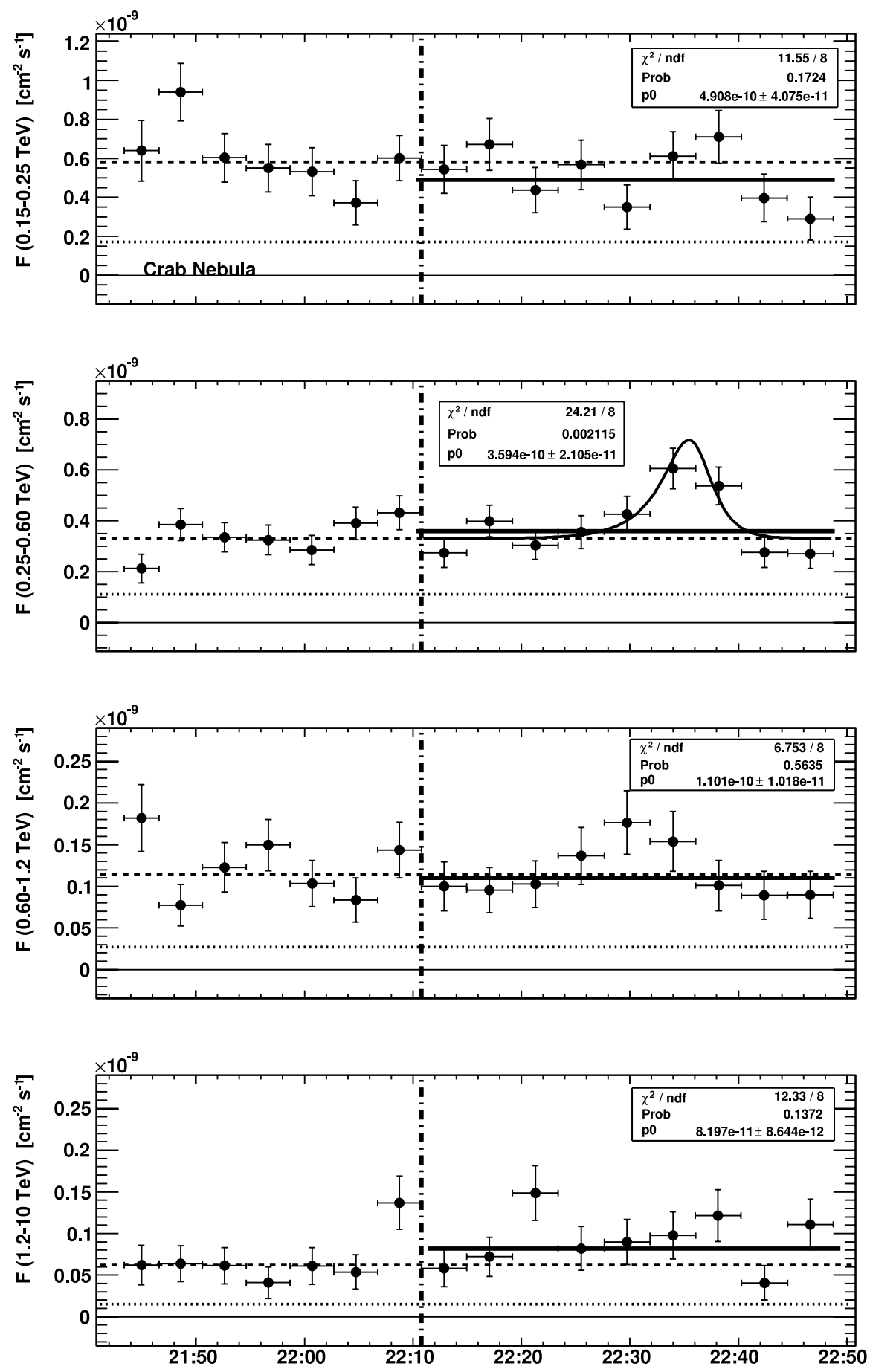

FIG. 6. - LC for the night June 30 with a time binning of 4 minutes, and separated in different energy bands, from top to bottom, $0.15-0.25,0.25-0.6,0.6-1.2$, and $1.2-10 \mathrm{TeV}$. The vertical bars denote $1 \sigma$ statistical uncertainties. For comparison, the Crab emission is also shown as a dotted horizontal line. The vertical dot-dashed line divides the data into stable (i.e., preburst) and variable (i.e., in-burst) emission. The horizontal dashed line represents the average of the stable emission. The variable (inburst) emissions of all energy ranges were fitted with a constant line. The results of the fits are given in the insets. The constant line fit on the energy range $0.25-0.6 \mathrm{TeV}$ was not satisfactory (see inset); yet this LC could be fitted with the flare model described by eq. (2) (see Table 3 for the resulting parameters). [See the electronic edition of the Journal for a color version of this figure.]

the energy range $0.25-0.6 \mathrm{TeV}$ shows a clear flux variation; a constant line fit gave $\chi^{2} / \mathrm{NDF}=24.2 / 8\left(P=2 \times 10^{-3}\right)$. The other energy ranges are compatible with a constant line fit, showing only a slight overall flux level variation with respect to the LC in the stable part. Therefore, if there is a flux variation, it is too small to be significantly seen in our data. Note that, among the four energy ranges used, the $0.25-0.60 \mathrm{GeV}$ energy range is the one with the highest sensitivity for flux variations. The variable LC from the energy range $0.25-0.60 \mathrm{TeV}$ was then fitted using equation (2), fixing $t_{0}$ to the time of the highest point in the LC. The resulting parameters of the fit are reported in Table 3; the rise/fall flux-doubling times are comparable to those obtained using the integrated LC above $0.15 \mathrm{TeV}$.

The same exercise on the flare July 9 gave a significantly different result, as shown in Figure 7. The flare is visible essentially in all energy ranges. In order to study possible time shifts between 
TABLE 3

Flare Model Parameters for June 30: Differential Emission

\begin{tabular}{|c|c|c|c|c|c|c|c|}
\hline $\begin{array}{l}\text { Energy Range } \\
\quad(\mathrm{TeV})\end{array}$ & $\begin{array}{c}a^{\mathrm{a}} \\
\left(10^{-10} \text { photons } \mathrm{cm}^{-2} \mathrm{~s}^{-1}\right)\end{array}$ & $\begin{array}{c}a \\
\text { (crab) }\end{array}$ & $\begin{array}{c}b \\
\left(10^{-10} \text { photons } \mathrm{cm}^{-2} \mathrm{~s}^{-1}\right)\end{array}$ & $\begin{array}{c}c \\
(\mathrm{~s})\end{array}$ & $\begin{array}{l}d \\
(\mathrm{~s})\end{array}$ & $\chi^{2} / \mathrm{NDF}^{\mathrm{b}}$ & $\begin{array}{l}P^{\mathrm{c}} \\
(\%)\end{array}$ \\
\hline $0.25-0.6 .$. & $3.30 \pm 0.23$ & $3.0 \pm 0.2$ & $7.5 \pm 2.8$ & $110 \pm 57$ & $61 \pm 26$ & $5.2 / 6$ & 51.8 \\
\hline
\end{tabular}

${ }^{\mathrm{a}}$ Integrated steady emission flux (left part of the graphs) in specified energy range.

${ }^{b}$ The $\chi^{2}$ value and number of degrees of freedom of the fit with eq. (2).

${ }^{c}$ Chance probability of having larger $\chi^{2}$ values.
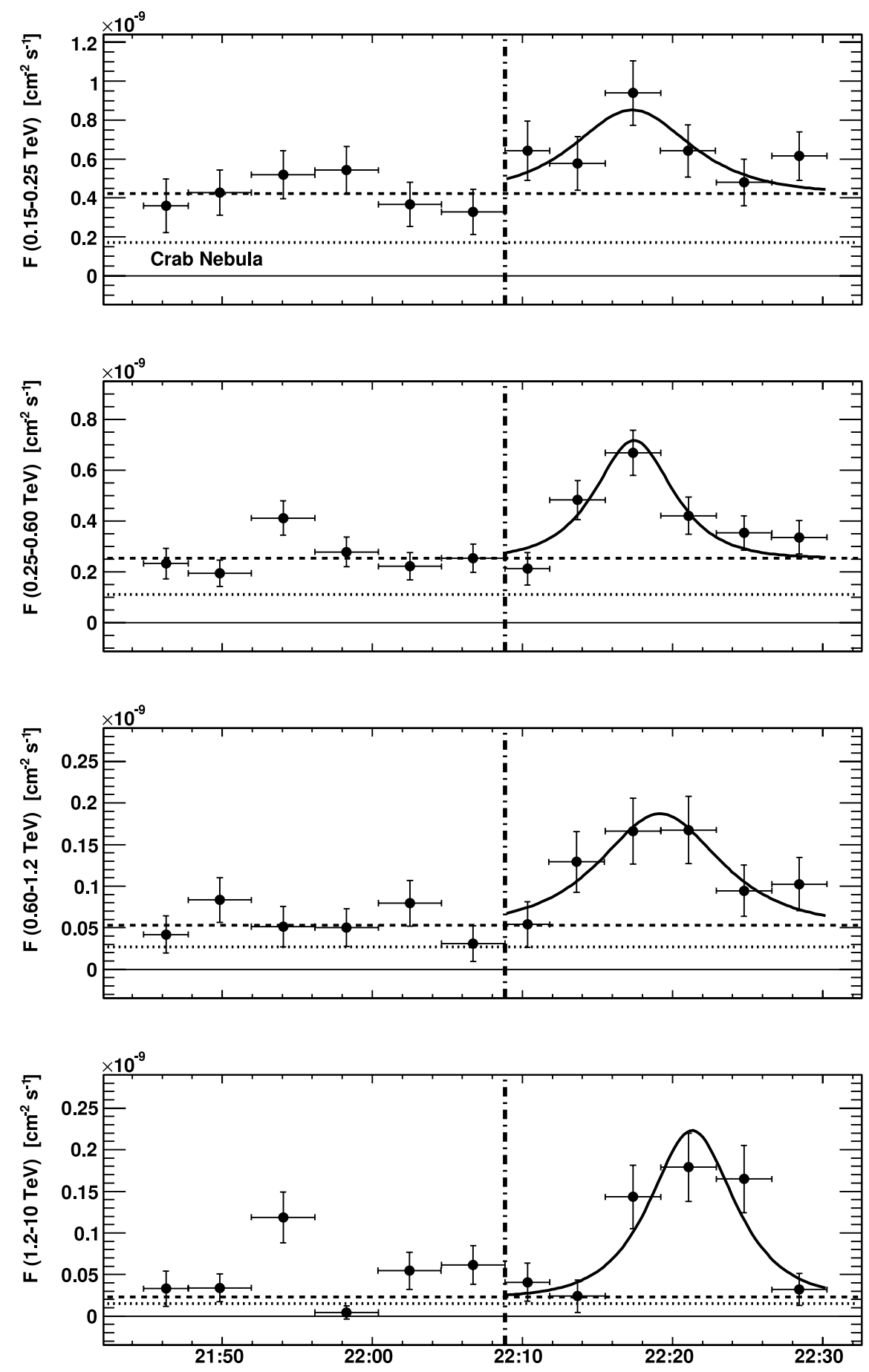

Fig. 7. - LC for the night July 9 with a time binning of 4 minutes, and separated in different energy bands, from top to bottom, $0.15-0.25,0.25-0.6,0.6-1.2$, and $1.2-10 \mathrm{TeV}$. The vertical bars denote $1 \sigma$ statistical uncertainties. For comparison, the Crab emission is also shown as a dotted horizontal line. The vertical dot-dashed line divides the data into stable (i.e., preburst) and variable (i.e., in-burst) emission. The horizontal dashed line represents the average of the stable emission. The variable (inburst) emissions of all energy ranges were fitted with a flare model described by eq. (2), where $c=d$ (rise=fall time). All parameters were left free in the fit. All LCs were considered simultaneously in the fit (combined fit). The resulting parameters from this combined fit are reported in Table 4. [See the electronic edition of the Journal for a color version of this figure.] 
TABLE 4

Flare Model Parameters for July 9 Resulting from a Combined Fit to All LCs from Figure 7 using Equation $(2)$ with $c=d$

\begin{tabular}{|c|c|c|c|c|c|}
\hline $\begin{array}{l}\text { Energy Range } \\
\quad(\mathrm{TeV})\end{array}$ & $\begin{array}{c}a^{\mathrm{a}} \\
\left(10^{-10} \text { photons } \mathrm{cm}^{-2} \mathrm{~s}^{-1}\right)\end{array}$ & $\begin{array}{c}a \\
(\mathrm{crab})\end{array}$ & $\begin{array}{c}b \\
\left(10^{-10} \text { photons } \mathrm{cm}^{-2} \mathrm{~s}^{-1}\right)\end{array}$ & $\begin{array}{l}c \\
(\mathrm{~s})\end{array}$ & $\begin{array}{c}t_{0}-t_{0}^{\mathrm{LC},} 0.15-0.25 \mathrm{TeVb} \\
\text { (s) }\end{array}$ \\
\hline $0.15-0.25 \ldots \ldots$ & $4.23 \pm 0.49$ & $2.48 \pm 0.28$ & $8.6 \pm 3.7$ & $143 \pm 92$ & $0 \pm 68$ \\
\hline 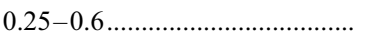 & $2.55 \pm 0.24$ & $2.32 \pm 0.09$ & $9.3 \pm 2.5$ & $95 \pm 28$ & $7 \pm 36$ \\
\hline 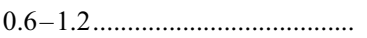 & $0.53 \pm 0.10$ & $1.96 \pm 0.37$ & $2.7 \pm 0.9$ & $146 \pm 56$ & $111 \pm 91$ \\
\hline $1.2-10$ & $0.23 \pm 0.06$ & $1.51 \pm 0.39$ & $4.0 \pm 0.9$ & $103 \pm 19$ & $239 \pm 40$ \\
\hline
\end{tabular}

Note. - The overall $\chi^{2} / \mathrm{NDF}=14.0 / 12(P=0.3)$.

a Integrated steady emission flux (left part of the graphs) in specified energy range.

b Parameter $t_{0}^{\mathrm{LC}, 0.15-0.25 \mathrm{TeV}}$ is the $t_{0}$ for the $\mathrm{LC}$ in the energy range $0.15-0.25 \mathrm{TeV}$. This is used as a reference value, and the error of this quantity is not taken into account.

the different energy ranges, we fit all LCs simultaneously (combined fit) with a flare model described by equation (2). In order to remove 1 degree of freedom and facilitate the fit procedure, we assume a symmetric flare with equal rise and fall flux-doubling times, that is, $c=d$ in equation (2). The resulting parameters from this combined fit are shown in Table 4 . The combined fit gave $\chi^{2} / \mathrm{NDF}=14.0 / 12(P=0.3)$, which implies that the measured flare is compatible with being symmetric. The rise/fall flux-doubling time is about 2 minutes for all the energy ranges. It is interesting to note that the position of the peak of the flare for the different LCs seems to vary somewhat with energy. The time difference between the highest energy range and the lowest energy range is $239 \pm 78 \mathrm{~s}$. If, instead, the energy range $0.25-0.6 \mathrm{TeV}$ is selected as the lowest energy range, which has a better defined flare (and thus a better determination of the peak position), the time difference is $232 \pm 54 \mathrm{~s}$.

In order to evaluate the significance of this time shift, we performed the same fit, but this time using a common $t_{0}$ for all LCs. The resulting fits are shown in Figure 8, and the resulting parameters from the fit in Table 5. The combined fit gave $\chi^{2} / \mathrm{NDF}=$ $26.6 / 15(P=0.04)$, which implies that such a situation is unlikely, and consequently that the time shift of $4 \pm 1$ minutes between the highest and the lowest energies is more probable.

Investigating the reliability of the time delay obtained from the combined fit, we performed a cross-correlation analysis on the LCs from July 9 with the methodology described in $\S 3.2$. For this study we used LCs with 2 minute time bins from the energy ranges $0.25-0.6$ and $1.2-10 \mathrm{TeV}^{38}$ The correlation coefficient and probability of correlation were computed after introducing time shifts of 2 minutes (one bin in the LCs). We obtained the highest values for a time lag of 4 minutes, which is consistent with the results from the combined fit shown above.

We want to point out that this is the first time that a possible time delay between flares at different energies is observed at VHE $\gamma$-ray energies, although such time lags have been detected for some $\mathrm{TeV}$ blazars at X-ray frequencies, viz., Mrk 421 (Ravasio et al. 2004) and PKS 2155-304 (Zhang et al. 2006a, 2006b). If the observed VHE time lag is assumed to be real, this suggests that we are observing the underlying dynamics of the relativistic electrons in both the synchrotron and IC emission, and the observation, therefore, supports SSC models.

It should be also noted that the relative amplitude of the flux variations observed in the LC for July 9 with respect to the baseline emission is significantly larger at the highest energies. This can be seen from the ratio $b / a$, where $a$ and $b$ are the coefficients in equation (2) describing, respectively, the baseline and ampli-

\footnotetext{
38 The flare observed in the LC from the energy range $0.15-0.25 \mathrm{TeV}$ is not very well defined because of the somewhat larger measurement errors and the smaller relative amplitude of the flux variation (with respect to the stable emission) with decreasing energy.
}

tude of the flare (see Table 4): $b / a=3.6 \pm 1.0$ and $17 \pm 4$ for, respectively, the $0.25-0.6$ and $1.2-10 \mathrm{TeV}$ bands. The July $9 \mathrm{LC}$ also shows some significant flux variation in its stable part: in the highest energy band, where activity is most conspicuous, a constant fit gives a $\chi^{2} / \mathrm{NDF}=20.6 / 5\left(P=9.6 \times 10^{-4}\right)$.

In summary, during the 2005 MAGIC observations of Mrk 501 we detected variability at VHE frequencies with flux-doubling times down to 2 minutes. This is about 50 times faster than the shortest previously observed variability times at VHE frequencies for Mrk 501 (Hayashida et al. 1998; Quinn et al. 1999; Aharonian et al. 1999a; Djanati-Ataï et al. 1999) and about 5 times shorter than the shortest observed variability for Mrk 421 (Gaidos et al. 1996). The above-presented flux variations are among the shortest ever observed in blazars (see also Aharonian et al. 2007). It is interesting to note that the Mrk 501 flux-doubling rise times observed by MAGIC in the VHE range are rather comparable to the shortest variability times observed at X-ray frequencies that were reported by Xue \& Cui (2005): a flare with a total duration of 15 minutes with a flux variation of $30 \%$. The authors, however, reported the presence of substructures, which point to the existence of variability on timescales shorter than 15 minutes. It is worth mentioning that for both X-ray and $\gamma$-ray the shortest flux variations occurred when the source was not in an exceptionally high emission state.

\subsection{Quantification of the Variability}

Mrk 501 has shown energy-dependent flux variations throughout the entire MAGIC observational campaign. We followed the description given in Vaughan et al. (2003) to quantify the flux variability by means of the fractional variability parameter $F_{\mathrm{var}}$, as a function of energy. In order to account for the individual flux measurement errors $\left(\sigma_{\mathrm{err}, i}\right)$, we used the "excess variance" (Nandra et al. 1997; Edelson et al. 2002) as an estimator of the intrinsic source variance. This is the variance after subtracting the expected contribution from measurement errors. For a given energy range, the $F_{\text {var }}$ is calculated as

$$
F_{\mathrm{var}}=\sqrt{\frac{S^{2}-\left\langle\sigma_{\mathrm{err}}^{2}\right\rangle}{\left\langle F_{\gamma}\right\rangle^{2}}}
$$

where $\left\langle F_{\gamma}\right\rangle$ is the mean photon flux, $S$ is the standard deviation of the $N$ flux points, and $\left\langle\sigma_{\text {err }}^{2}\right\rangle$ is the average mean square error, all determined for a given energy bin. The uncertainty on $F_{\mathrm{var}}$ is estimated according to

$$
\Delta F_{\mathrm{var}}=\sqrt{\left(\sqrt{\frac{1}{2 N}} \frac{\left\langle\sigma_{\mathrm{err}}^{2}\right\rangle}{\left\langle F_{\gamma}\right\rangle^{2} F_{\mathrm{var}}}\right)^{2}+\left(\sqrt{\frac{\left\langle\sigma_{\mathrm{err}}^{2}\right\rangle}{N}} \frac{1}{\left\langle F_{\gamma}\right\rangle}\right)^{2}} .
$$



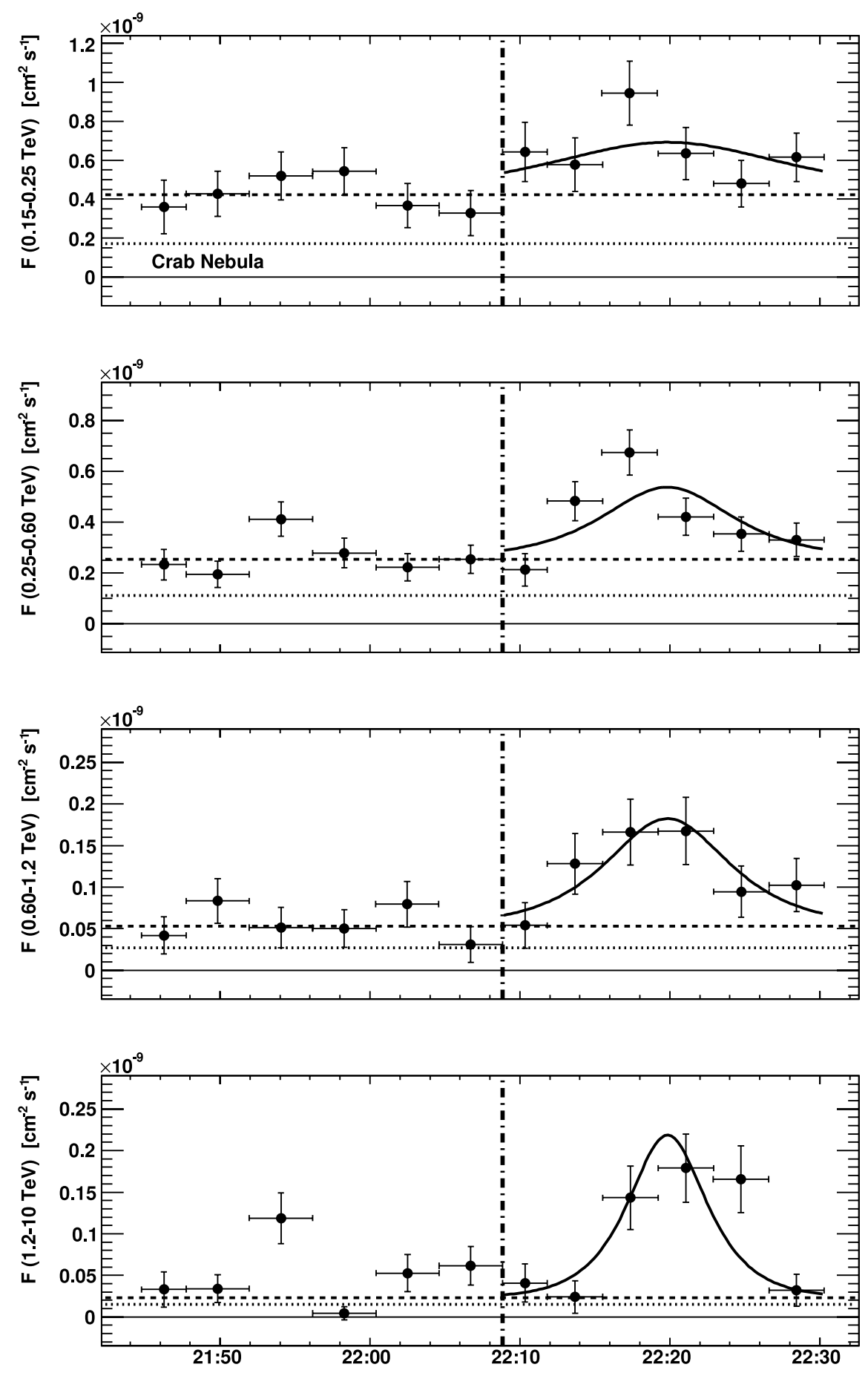

Fig. 8. - Same as in Fig. 7, but with a common $t_{0}$ (which was also fitted) for all LCs. The resulting parameters from this combined fit are reported in Table 5. [See the electronic edition of the Journal for a color version of this figure.]

Figure 9 shows the derived $F_{\text {var }}$ values for five logarithmic energy bins, spanning from 0.14 to $8 \mathrm{TeV}^{39}$ The left panel includes all data, while the right panel includes all data except for the two active nights. The result indicates a larger amplitude flux variation at higher energies, which is clearly discernible even when the active state data are excluded (the null probability being $P \sim 10^{-5}$ ).

Figure 10 shows the $F_{\text {var }}$ values as derived for the individual active nights of June 30 and July 9 . The flux variability in these

39 The $F_{\text {var }}$ is not meaningful below $0.14 \mathrm{TeV}$ (i.e., below the threshold energy of the instrument) because the flux errors are rather large, which makes $\left\langle\sigma_{\text {err }}^{2}\right\rangle \sim S^{2}$. nights is smaller than for the entire observational campaign, as one would expect from simple inspection of Figure 1. In the night of June $30, F_{\text {var }}$ does not increase significantly with energy. In contrast to June 30, there is a clear increase of $F_{\mathrm{var}}$ with energy in the night of July 9 , in spite of the larger error bars coming from a shorter observation time and a lower mean flux.

In summary, during the year 2005 MAGIC observations, the VHE $\gamma$-ray flux variability of Mrk 501 was found to significantly increase with energy, on timescales from months to less than an hour. A similar effect (on timescales $\gtrsim \frac{1}{2} \mathrm{hr}$ ) was also detected in X-rays in 1997, 1998, and 2000 (Gliozzi et al. 2006; based on RXTE data). Another X-ray evidence was found for the TeV blazar, PKS 
TABLE 5

Flare Model Parameters for July 9 Resulting from a Combined Fit to All lCs from Figure 8 Using Equation (2) with $c=d$, and with a Common $t_{0}$ For All LCs

\begin{tabular}{|c|c|c|c|c|c|}
\hline $\begin{array}{l}\text { Energy Range } \\
\quad(\mathrm{TeV})\end{array}$ & $\begin{array}{c}a^{\mathrm{a}} \\
\left(10^{-10} \text { photons } \mathrm{cm}^{-2} \mathrm{~s}^{-1}\right)\end{array}$ & $\begin{array}{c}a \\
(\mathrm{crab})\end{array}$ & $\begin{array}{c}b \\
\left(10^{-10} \text { photons } \mathrm{cm}^{-2} \mathrm{~s}^{-1}\right)\end{array}$ & $\begin{array}{c}c \\
(\mathrm{~s})\end{array}$ & $\begin{array}{c}t_{0}-t_{0}^{\mathrm{LC},}, 0.15-0.25 \mathrm{TeVb} \\
\text { (s) }\end{array}$ \\
\hline $0.15-0.25 \ldots \ldots \ldots \ldots \ldots \ldots \ldots \ldots \ldots \ldots$ & $4.23 \pm 0.49$ & $2.48 \pm 0.28$ & $5.4 \pm 2.2$ & $301 \pm 210$ & $0 \pm 42$ \\
\hline $0.6-1.2$ & $0.53 \pm 0.10$ & $1.96 \pm 0.37$ & $2.6 \pm 0.8$ & $153 \pm 56$ & $0 \pm 42$ \\
\hline $1.2-10$ & $0.23 \pm 0.06$ & $1.51 \pm 0.39$ & $3.9 \pm 1.0$ & $97 \pm 22$ & $0 \pm 42$ \\
\hline
\end{tabular}

Note.-The overall $\chi^{2} / \mathrm{NDF}=25.6 / 15(P=0.04)$.

${ }^{a}$ Integrated steady emission flux in specified energy range (left part of the graphs).

${ }^{\mathrm{b}}$ Parameter $t_{0}^{\mathrm{LC},}, 0.15-0.25 \mathrm{TeV}$ is the $t_{0}$ for the LC in the energy range $0.15-0.25 \mathrm{TeV}$. This is used as a reference value, and the error of this quantity is not taken into account.

2155-304 (Zhang et al. 2006b). The largest X-ray $F_{\text {var }}$ value in $\mathrm{X}$-rays for Mrk 501 was $\sim 0.6-0.7$ in the highest energy bin at 10$20 \mathrm{keV}$, and it was found in 1998 June and 2000 July-September (Gliozzi et al. 2006). In 1997, however, despite Mrk 501 showing the highest X-ray $(2-10 \mathrm{keV})$ fluxes from the last 10 years, the largest observed $F_{\text {var }}$ value in X-rays was only $\sim 0.4$. It is interesting to note that, in the VHE $\gamma$-ray range, we observe a maximum $F_{\text {var }}$ of $\gtrsim 0.6$, which increases to $\gtrsim 1.2$ when the two active nights are included. This suggests that Mrk 501 is more variable in VHE $\gamma$-rays than in X-rays.

\section{VHE SPECTRA}

The differential photon spectra of Mrk 501 were parameterized with a simple PL function:

$$
\frac{d F}{d E}=K_{0}\left(\frac{E}{0.3 \mathrm{TeV}}\right)^{-a},
$$

where $K_{0}$ is a normalization factor and $a$ is the photon index. The MAGIC sensitivity permits us to derive VHE spectra of Mrk 501 on a daily basis, independent of its flux level, a real asset for unbiased precision studies of blazars. The PL spectral parameters for each single night are reported in Table 1 .

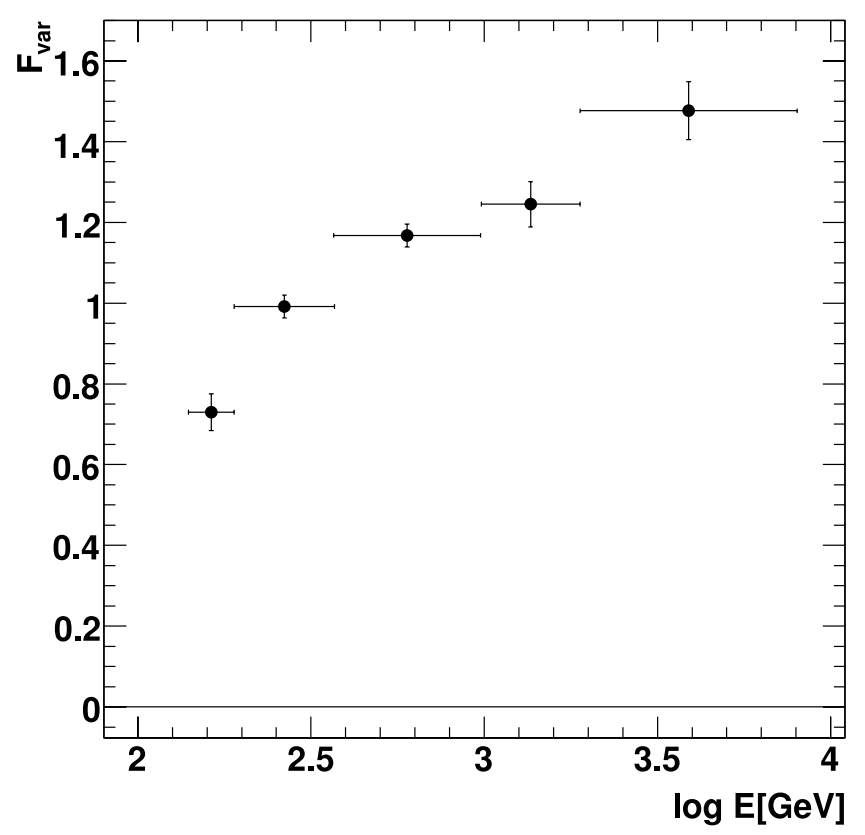

During our observations, the VHE emission of Mrk 501 showed a very dynamic behavior, with significant spectral variability on a timescale of days (see $\S \S 4.1$ and 4.2). Nevertheless, most of the data are well described by the simple PL function of equation (6). This does not hold for the two flaring nights of June 30 and July 9 , which are therefore discussed separately in $\S \S 4.3$ and 4.4.

\subsection{Spectral Index versus Flux}

The Mrk 501 VHE spectrum was measured on a night-bynight basis, which allowed investigating possible correlations between the PL spectral index and intensity, as shown in Figure 11. The data of June 30 and July 9 were again split into the stable and variable parts (see Fig. 5), and consequently, Figure 11 contains 26 instead of 24 points. The data points are well described by a linear fit, but a constant is clearly excluded $\left(\chi^{2} / \mathrm{NDF}=74.7 / 25\right.$, i.e., $\left.P=7.4 \times 10^{-7}\right)$. On average, the Mrk 501 spectrum hardens when the emission increases. Such a correlation was already reported by Pian et al. (1998) and Tavecchio et al. (2001), although on substantially longer timescales.

In order to test the Ansatz of a linear correlation in spectral index versus $\gamma$-ray flux (see Fig. 11), we applied a correlation analysis as described in $\S 3.2$. The linear fit indicates anticorrelation $\left(\chi^{2} / \mathrm{NDF}=19.65 / 24, P=0.72\right)$. The correlation factor is -0.48 ,

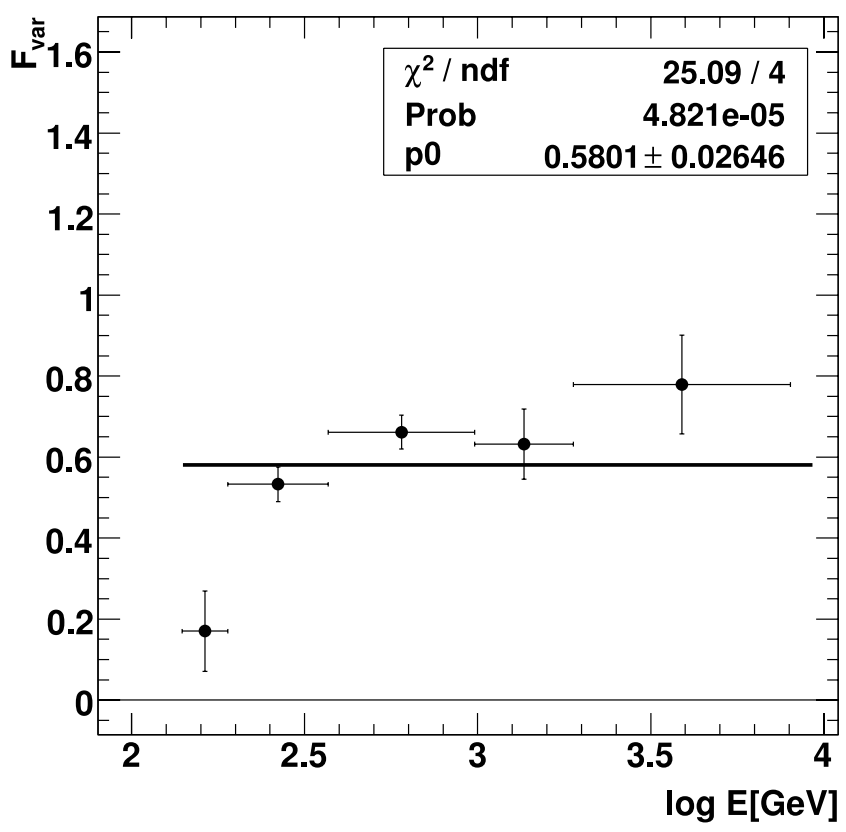

FIG. 9.-Fractional variability parameter as derived for five energy bins. Vertical bars denote $1 \sigma$ uncertainties; horizontal bars indicate the width of each energy bin. The left panel includes all data. The right panel includes all but the June 30 and July 9 data. The horizontal line in the right panel results from a constant fit to the data points (see inset for fit parameters). [See the electronic edition of the Journal for a color version of this figure.] 

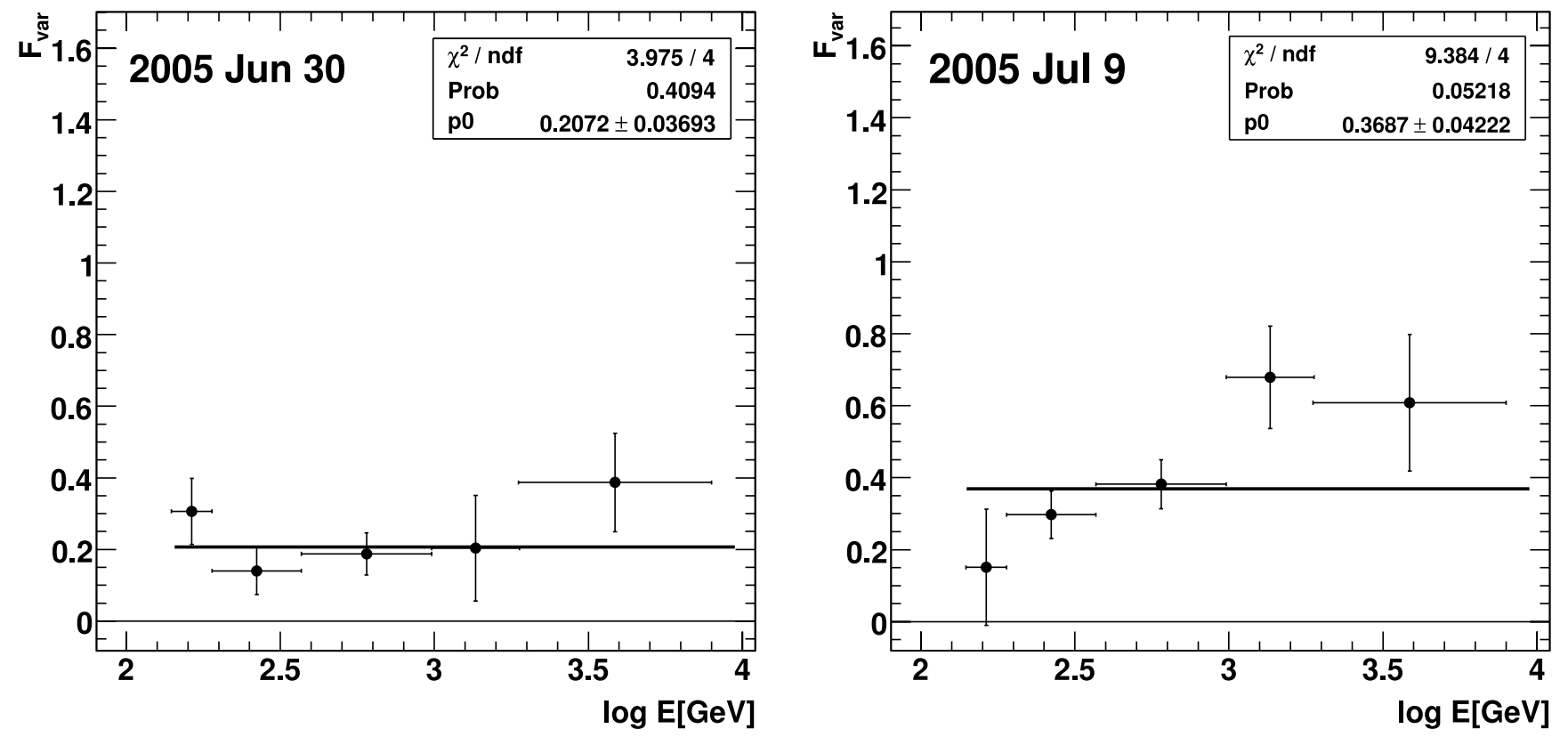

FIG. 10. - Fractional variability parameter as computed for the nights of June 30 and July 9. Vertical bars denote $1 \sigma$ uncertainties, horizontal bars indicate the width of each energy bin. The horizontal lines are the result of a constant fit to the data points (see inset for fit parameters). [See the electronic edition of the Journal for a color version of this figure.]

and the corresponding probability distributions are shown in Figure 12 . We find the probability $P_{\alpha, \gamma}\left(f_{A}, f_{B}\right)=0.35$ to be higher than $P_{\alpha, \gamma}\left(f_{A}, f_{C}\right)=0.12$, which supports a modest anticorrelation.

Similarly, the probability for the first scenario to be true and for the second to be false is $P_{\alpha, \gamma}\left(f_{A}, f_{B}\right)\left[1-P_{\alpha, \gamma}\left(f_{A}, f_{C}\right)\right]=$ 0.30 , while the probability for the opposite case is $[1-$ $\left.P_{\alpha, \gamma}\left(f_{A}, f_{B}\right)\right] P_{\alpha, \gamma}\left(f_{A}, f_{C}\right)=0.08$. In conclusion, this correlation study indicates a spectral hardening with increased flux.

\subsection{Spectra at Different Flux Levels}

In order to investigate the spectra at different flux levels, the diurnal data were combined into three groups, depending on whether the integral flux above $0.15 \mathrm{TeV}, F_{0.15 \mathrm{TeV}}$ (measured in crab), was low $\left(F_{0.15 \mathrm{TeV}}<0.5 \mathrm{crab}\right)$, medium $\left(0.5 \mathrm{crab}<F_{0.15 \mathrm{TeV}}<\right.$ $1.0 \mathrm{crab})$, or high $\left(1.0 \mathrm{crab}<F_{0.15 \mathrm{TeV}}\right)$. Based on the chosen flux limits, the low-, medium- and high-flux data sets consist of 12, eight, and two nights, respectively (see Table 1 for detailed statistics). The data from June 30 and July 9 are discussed in $\S 4.3$ and are not included in the analysis here. The differential photon spectrum for all three flux regions together with the PL fit results

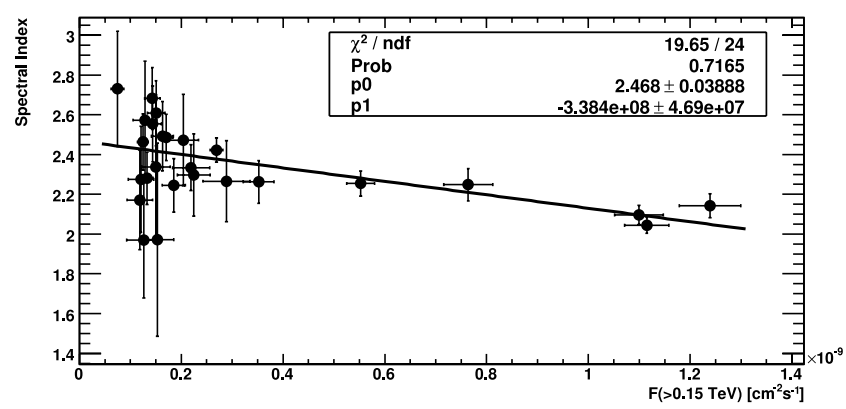

FIG. 11.-Correlation between spectral shape and integrated flux above $0.15 \mathrm{TeV}$. Each point denotes a single night of observation. The error bars denote $1 \sigma$ uncertainties. The June 30 and July 9 data were split chronologically into two data sets each, corresponding to the preflare (stable) and in-flare (variable) emission shown in Fig. 5. [See the electronic edition of the Journal for a color version of this figure.] are shown in Figure 13; the fit parameters are also listed in Table 6. Even with such a simple parameterization, our data do suggest a spectral hardening with increasing flux. The results of this analysis are consistent with the trend seen in Figure 11 and discussed in $\S 4.1$.

The HEGRA CT system measured the spectra of Mrk 501 in 1998-1999, at a time when its flux level was substantially below the one of the Crab ( $\left.\sim \frac{1}{3} \mathrm{crab}\right)$. The observation covered $122 \mathrm{hr}$ (Aharonian et al. 2001). The reported spectrum could be fitted with a PL in the energy range $0.5-10 \mathrm{TeV}$, giving a spectral index of $2.76 \pm 0.08 .{ }^{40}$ It is interesting to note that this spectral index is slightly softer than the $2.45 \pm 0.07$ we obtained for the low flux ( $17 \mathrm{hr}$ of observation, $\sim 0.4 \mathrm{crab}$ ) in the energy range $0.1-6 \mathrm{TeV}$. This spectral shape difference might be caused by a

40 An exponential cutoff of $\sim 5 \mathrm{TeV}$ was suggested in Aharonian et al. (2001) although the experimental data are perfectly compatible with both hypotheses, the simple PL $\left(\chi^{2} / \mathrm{NDF}=12.9 / 14 ; P=0.53\right)$ and the PL with exponential cutoff $\left(\chi^{2} / \mathrm{NDF}=9.2 / 13 ; P=76\right)$.

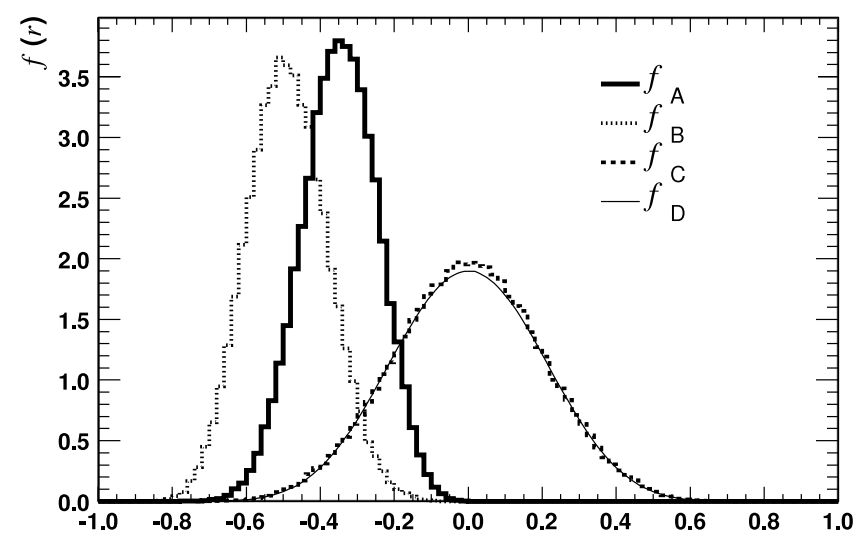

FIG. 12.- The pdf's of the correlation coefficient between spectral index and $\gamma$-ray fluxes: $f_{\mathrm{A}}$ data, $f_{\mathrm{B}}$ perfectly correlated case, $f_{\mathrm{C}}$ uncorrelated case, and $f_{\mathrm{D}}$ analytical solution in the uncorrelated case. See text for further details. [See the electronic edition of the Journal for a color version of this figure.] 


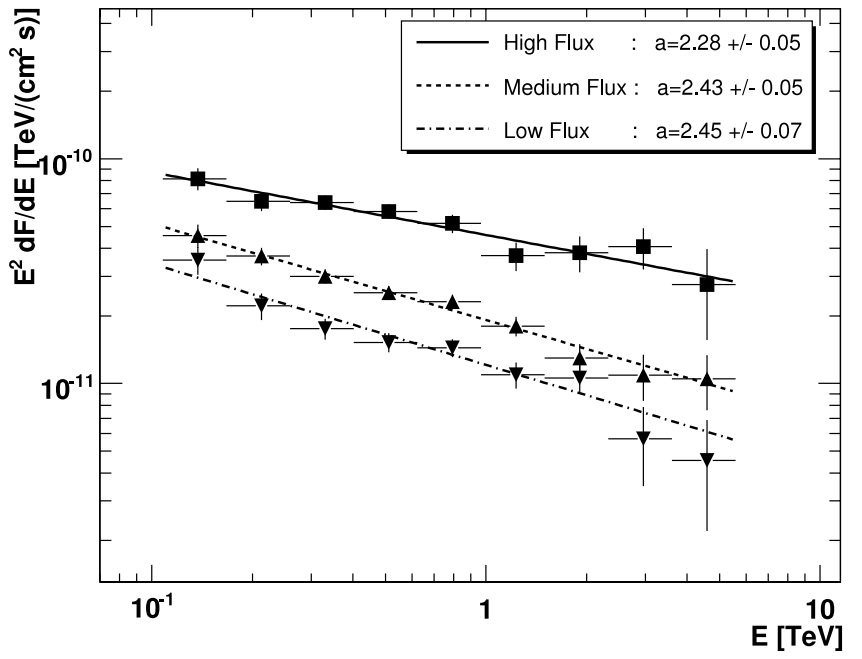

FIG. 13.- Measured spectra of Mrk 501 for three different $>0.15 \mathrm{TeV}$ flux levels: low (downward-pointing triangles), medium (upward-pointing triangles), and high (squares). Vertical bars denote $1 \sigma$ uncertainties; horizontal bars denote energy bins. Lines show PL best fits. See Table 6 for fit parameters. [See the electronic edition of the Journal for a color version of this figure.]

possible softening of the spectra above 1-2 TeV. Note that the fits to the spectra measured by MAGIC are mostly constrained by the points below $2 \mathrm{TeV}$ (due to low photon statistics at the highest energies), while the fits to the spectra measured by HEGRA are mostly constrained by the points above $1 \mathrm{TeV}$. Certainly, a factor contributing to the softening of the spectra at the highest energies is the $\gamma$-ray extinction due to pair production by interaction with the EBL. According to Kneiske et al. (2004), the attenuation of $\gamma$-rays coming from Mrk 501 is $\sim 30 \%$ at $1 \mathrm{TeV}$ and $\sim 50 \%$ at $10 \mathrm{TeV}$, while it is $\leq 15 \%$ for energies below $0.5 \mathrm{TeV}$.

\subsection{Spectra during Active Nights}

The differential photon spectra from June 30 and July 9 were fitted with the simple PL described in equation (5), as well as with the log-parabolic function:

$$
\frac{d F}{d E}=K_{0}\left(\frac{E}{0.3 \mathrm{TeV}}\right)^{-a-b \log _{10}(E / 0.3 \mathrm{TeV})} .
$$

Here $K_{0}$ is a normalization factor, $a$ is the spectral index at $0.3 \mathrm{TeV}$, and $b$ is a curvature parameter (for $b>0$ the spectrum hardens/ softens at energies below/above $0.3 \mathrm{TeV}$ ). The log-parabola is a simple function to describe curved spectra and, as pointed out by Massaro et al. (2004, 2006), can be directly related to the intrinsic physical processes occurring in the source. The results of both the $\mathrm{PL}$ and the log-parabolic functions are reported in Table 7. Since the log-parabolic fit describes the data more accurately than the PL fit, this suggests that the differential photon spectra of the two flaring nights are curved. The spectra and their fits are shown in Figure 14. Note that the peak of the energy spectrum is located in the covered energy range in both nights.

The peak location in a spectrum described by equation (6) is given by

$$
E_{\text {peak }}=\left(10^{(2-a) / 2 b}\right) 0.3 \mathrm{TeV},
$$

with an associated uncertainty of

$$
\Delta E_{\text {peak }}=E_{\text {peak }} \frac{\ln 10}{2 b} \sqrt{V_{a a}+V_{b b}\left(\frac{a-2}{b}\right)^{2}-2 V_{a b}\left(\frac{a-2}{b}\right)},
$$

where $V_{a a}, V_{b b}$, and $V_{a b}$ are the coefficients of the covariance matrix. Using these equations, one finds that the peak locations are $0.43 \pm 0.06$ and $0.25 \pm 0.07 \mathrm{TeV}$ for the spectra measured during June 30 and July 9 , respectively. It should be noted that these spectra are not corrected for EBL absorption and are therefore not intrinsic to Mrk 501. After correction for EBL absorption, the spectral peaks are shifted toward higher energies (see $\S 5.5)$.

The detection of a spectral curvature and measurement of the peak location were first reported by the Whipple and CAT collaborations (Samuelson et al. 1998; Djanati-Ataï et al. 1999; Piron 2000, 2003) based on 1997 Mrk 501 data. However, in those studies the spectra were not corrected for the EBL absorption. Such correction is relevant because the measured curvature in those energy spectra occurred essentially above $1-2 \mathrm{TeV}$, where current EBL models predict a $\geq 40 \%$ attenuation. Below $1 \mathrm{TeV}$, little (if at all) curvature could be seen within the quoted $1 \sigma$ statistical errors. Therefore, we suggest that the curvature and peak location reported in those studies may be significantly affected (if not dominated) by the EBL absorption. On the other hand, the spectral curvature in the MAGIC data is dominated by points below $1 \mathrm{TeV}$, since the higher energy points hardly constrain the fit due to their substantially larger error bars. The curvature we measure thus is affected, but not dominated by EBL absorption.

TABLE 6

Stacked Analysis: Mean Spectral Parameters

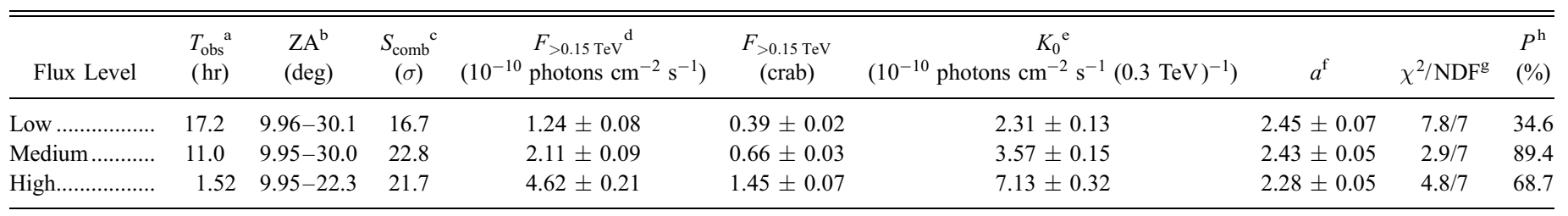

NoTE.-See $\S 4.2$ for definition of flux levels.

${ }^{a}$ Net observation time after removing bad-quality runs.

b Zenith angle range covered during the observation.

c Combined significance of detected signal in energy range $0.10-10 \mathrm{TeV}$.

d Integrated flux above $0.15 \mathrm{TeV}$.

e Normalization factor of the PL fit.

${ }^{f}$ Slope of the PL fit.

g The $\chi^{2}$ value and number of degrees of freedom of the PL fit.

h Chance probability of having $\chi^{2}$ values. 
TABLE 7

PL (eq. [5]) and Log-Parabolic (eq. [6]) Fit Results for June 30 and July 9: Night-integrated Spectra

\begin{tabular}{|c|c|c|c|c|c|c|c|c|c|}
\hline Date & \multicolumn{4}{|c|}{ Fit Performed with Equation (5) } & \multicolumn{5}{|c|}{ Fit Performed with Equation (6) } \\
\hline Jun 30 & $17.4 \pm 0.05$ & $2.09 \pm 0.03$ & $26.1 / 11$ & 0.6 & $18.6 \pm 0.06$ & $1.89 \pm 0.06$ & $0.35 \pm 0.09$ & $6.1 / 10$ & 80.1 \\
\hline Jul 9....................... & $14.3 \pm 0.06$ & $2.20 \pm 0.04$ & $22.5 / 11$ & 2.1 & $15.5 \pm 0.07$ & $2.06 \pm 0.07$ & $0.36 \pm 0.16$ & $15.2 / 10$ & 12.5 \\
\hline
\end{tabular}

${ }^{\text {a }}$ Chance probability for larger $\chi^{2}$ values.

\subsection{Intranight Spectral Variations}

As discussed in $\S 3.3$, during the active nights of June 30 and July 9 the VHE emission of Mrk 501 can be divided into a stable (preburst) and variable (in-burst) part. In order to study potential changes in the spectral shape, we derived the differential photon spectrum for the two parts of each night (Fig. 5). Since each spectrum is based on $\leq \frac{1}{2} \mathrm{hr}$ exposure, this procedure certainly increases the statistical errors. The four spectra were fitted with the log-parabolic function in equation (6), which is preferred over the simple PL function (see $\S 4.3$ ). The results of the fit, as well as other relevant information (e.g., net observing time, significance of the signal, goodness of fit), are reported in Table 8 . The spectra and the corresponding fits are plotted in Figure 15. In both nights, there is marginal $(1 \sigma)$ evidence for a spectral hardening during the flare.

We also studied the time evolution of the hardness ratio, defined as the ratio $F(1.2-10 \mathrm{TeV}) / F(0.25-1.2 \mathrm{TeV})$ and which is computed directly from the LCs shown in Figures 6 and 7. The resulting graph is shown in Figure 16. The hardness ratios for the preburst and in-burst part are quantified by means of a constant fit. In both nights the hardness ratio is somewhat larger $(1-2 \sigma)$ in the in-burst than in the preburst part, in agreement with the observed spectral hardening (see Fig. 15). It is worth noting that the hardness ratios for the preburst and in-burst time windows of June 30 are statistically compatible with being constant, while those of July 9 are much less so, as shown in the insets of Figure 16.

The evolution of the hardness ratio with the emitted flux above $0.25 \mathrm{TeV}$ is shown in Figure 17. Both nights show some evidence for a larger spread in the in-burst part than in the preburst part. The evolution of the in-burst points from June 30, however, is somewhat chaotic, while the evolution of the in-burst points from

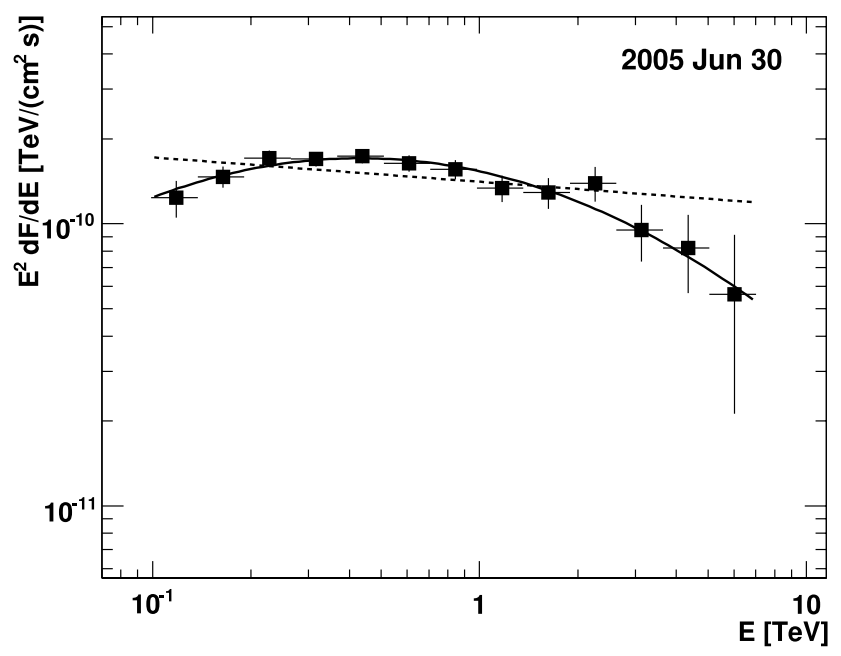

July 9 shows a clear loop pattern rotating counterclockwise. The physical interpretation of this feature is given in $\S 5.3$. Concluding, a spectral hardening with increased emission characterizes the VHE emission of Mrk 501 also at short timescales.

\section{DISCUSSION}

In this section we discuss the VHE LC of Mrk 501 for 2005 May-July in comparison to previous IACT observations made from the years 1997 through 2000. We also discuss the observed rapid flux variability in the framework of a basic model of gradual electron acceleration. The broadband spectral features of Mrk 501 and the intrinsic VHE spectra from different activity states are discussed in the framework of a basic SSC model. We also model the ensuing intrinsic VHE spectra, corresponding to different activity states, in an SSC framework.

\subsection{Historical Light Curve}

It is interesting to examine Mrk 501's VHE activity in 2005, as measured by MAGIC in perspective to that recorded in 19972000 by other IACTs such as HEGRA CT1 (Kranich 2000), HEGRA CT System (Multi-Messenger Group 2006 ${ }^{41}$ ), Whipple (Quinn et al. 1999), and CAT (Djanati-Ataï et al. 1999; Piron 2003). The long-term Mrk 501 LC covering the years $1997-$ 2005 is shown in Figure 18. For easier comparison among instruments covering different energy ranges, the integral flux values are given in crab. The mean fluxes for each year and instrument are shown in the insets of Figure 18. They were derived using the

${ }^{41}$ Available at http://www-zeuthen.desy.de/multi-messenger/GammaRayData/ index.html.

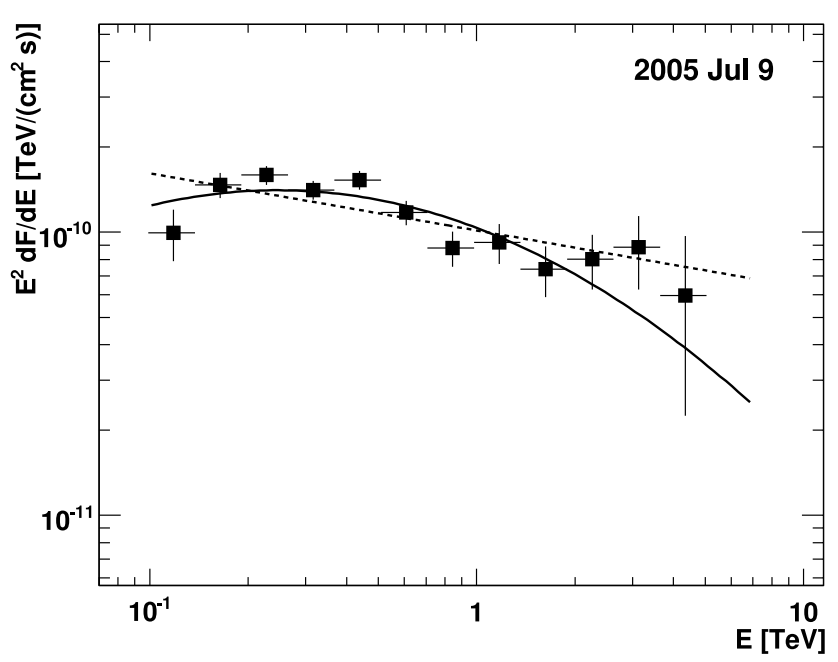

Fig. 14.- Measured spectra for the nights of June 30 and July 9 when Mrk 501 flared. Bars are as in Fig. 13. Spectral fits are a PL (dashed line; see eq. [5]) and a logparabolic function (solid line; see eq. [6]). See Table 7 for fit parameters. [See the electronic edition of the Journal for a color version of this figure.] 
TABLE 8

Log-Parabolic (eq. [6]) Fit Results for 2005 June 30 and July 9: Preburst and Burst Spectra

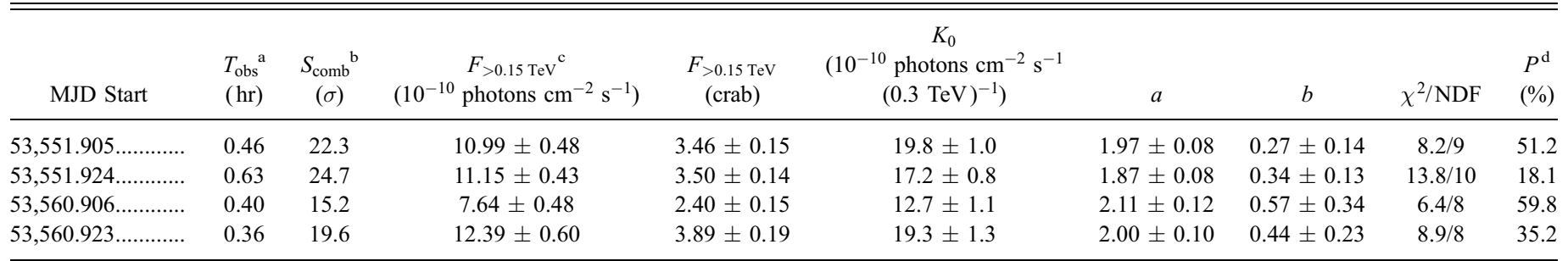

${ }^{a}$ Net observation time after removing bad-quality runs.

${ }^{\mathrm{b}}$ Combined significance of detected signal in energy range $0.1-10 \mathrm{TeV}$.

c Integrated $\gamma$-ray flux above $0.15 \mathrm{TeV}$.

${ }^{\mathrm{d}}$ Chance probability for larger $\chi^{2}$ values.

exposure times as statistical weights (whenever this information was available) and excluding data points $>3 \sigma$ away from each mean value, to permit a better comparison. ${ }^{42}$ For MAGIC, mean fluxes were computed in the other IACTs' energy bands. In 1997, when Mrk 501 was much brighter than the Crab, Whipple and CAT fluxes were in mutual agreement, but they significantly differed from the HEGRA CT System and HEGRA CT1 fluxes, the reasons being (1) the highest flux nights (1997 May-July) happened to be covered by HEGRA CT and CT1 but not by Whipple and CAT and (2) the highest threshold energies of HEGRA CT and CT1, together with the fact that when Mrk 501 is active its spectrum becomes significantly harder than the Crab's (see $\S \S 4.1$ and 4.2), hence fluxes measured in crab are larger at higher energies. In 1998 and 1999 the various IACT data agreed on a flux of $\sim 0.15-0.20 \mathrm{crab}$, i.e., an order of magnitude lower than in 1997. In 2000 March through May, the mean VHE flux of Mrk 501 increased to a level of $0.35 \pm 0.09$ (HEGRA CT1) and $1.19 \pm$ 0.17 (Whipple), the main reason for the discrepancy being that HEGRA CT1 missed the nights with highest flux measured by Whipple. In 2005 May through July, the mean baseline VHE flux from Mrk 501 was $\sim 0.5$ crab, significantly lower than in 1997 and 2000, but higher than in 1998 and 1999.

\footnotetext{
${ }^{42}$ Some HEGRA CT1, Whipple, and CAT fluxes are negative, unsurprisingly because many of the fluxes from these instruments are just $\leqslant 2 \sigma$ measurements. For the HEGRA CT System we have no information about negative values; hence, the corresponding mean fluxes may be slightly overestimated.
}

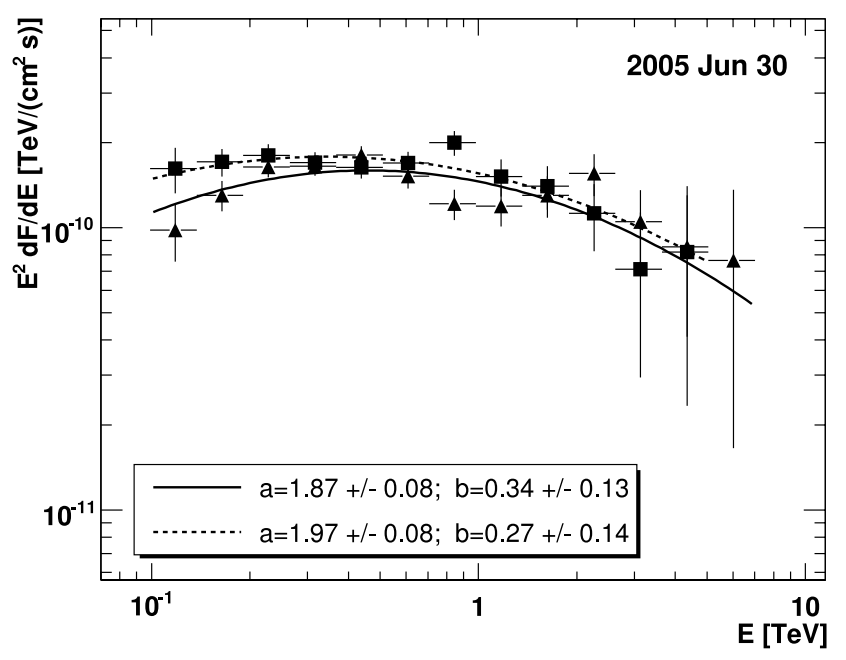

A 23 day flux periodicity was claimed by Kranich (2000) using data from HEGRA CT1. Recently, Osone (2006) confirmed the 23 day periodicity at VHE frequencies and extended it to $\mathrm{X}$-rays (based on RXTE ASM data) using a more sophisticated time analysis. This periodic modulation in the VHE emission of Mrk 501 may be evidence of a binary black hole system with separation of the order of the gravitational radius, as suggested by Rieger \& Mannheim $(2000,2003)$, or selective absorption of $\gamma$-rays in the radiation of a hot spot orbiting in the inner part of the accretion disk, as pointed out by Bednarek \& Protheroe (1997b). This periodicity was not seen in the 1998-2000 campaigns, when the source was apparently not very active. This might indicate that such a periodicity in the emitted flux occurs only when the source shows very high activity. The MAGIC 2005 data did not have the required coverage for such a timing analysis.

\subsection{Interpretation of the Measured Fast Flux Variations and Energy-dependent Time Delays}

The very short flux-doubling time and the energy-dependent time delays of the flux variations can give us information on the acceleration processes occurring in Mrk 501. In this section we argue that gradual electron acceleration in the emitting plasma can provide a natural explanation of the observed time structures:

1. Flare decay timescale.- - Let us assume that the maximum energies of electrons accelerated in the relativistic blob are determined by their radiation energy losses on synchrotron and IC

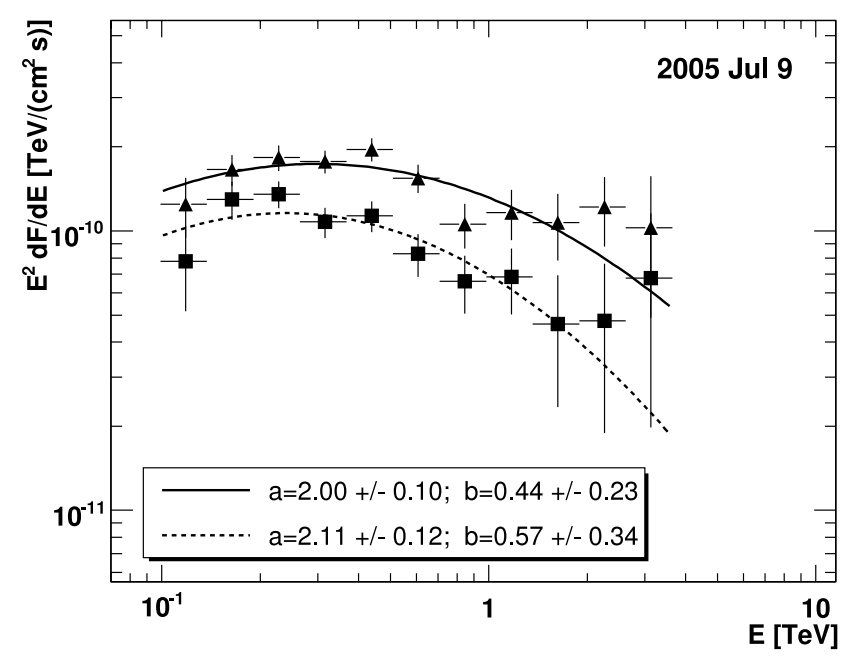

FIG. 15.- Spectra of Mrk 501 in the nights of June 30 and July 9 corresponding to the preburst (stable) and in-burst (variable) emission (see $\S 3.3$ and Fig. 5). Squares/ triangles denote stable/variable emission. Bars are as in Fig. 13. The insets show the log-parabolic fit parameters (see eq. [6]). [See the electronic edition of the Journal for a color version of this figure.] 

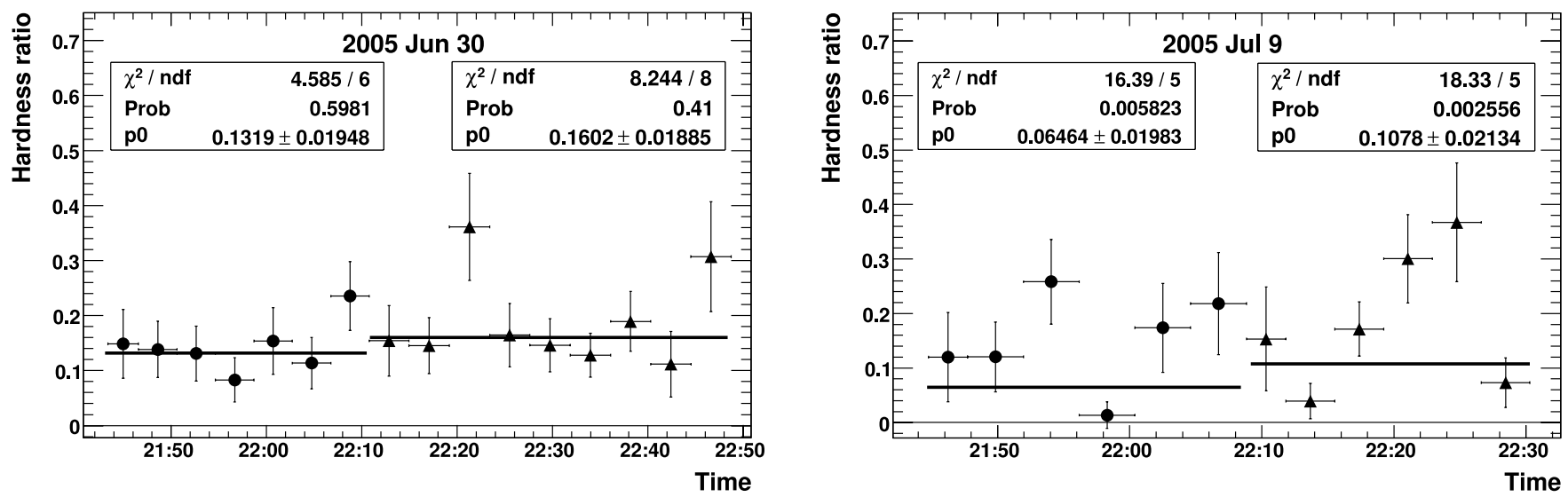

Fig. 16. - Hardness ratio $F(1.2-10 \mathrm{TeV}) / F(0.25-1.2 \mathrm{TeV})$ vs. time for the nights of June 30 and July 9 . Horizontal bars represent the 4 minute time bins, and vertical bars denote $1 \sigma$ statistical uncertainties. Squares and triangles denote preburst (stable) and in-burst (variable) emission, respectively (see $\S 3.3$ and Fig. 5). The lines result from a constant fit to the data points (see insets for fit parameters). [See the electronic edition of the Journal for a color version of this figure.]

processes, as expected in the SSC model. The acceleration time should then equal the energy loss timescale

$$
\tau_{\mathrm{acc}}=\tau_{\mathrm{cool}}
$$

where

$$
\tau_{\mathrm{acc}}=\frac{E_{e}^{\prime}}{\dot{P}_{\mathrm{acc}}}=\frac{E_{e}^{\prime}}{\xi c E_{e}^{\prime} / R_{\mathrm{L}}} \approx \frac{0.1 E_{e}^{\prime}}{\xi B} \mathrm{~s} .
$$

Here $E_{e}^{\prime}$ is the electron energy (in TeV) in the blob, $\dot{P}_{\text {acc }}$ is the rate of energy gain during the acceleration process, $B$ (in $\mathrm{G}$ ) is the magnetic field strength in the acceleration region, $R_{\mathrm{L}}$ is the electron Larmor radius, and $\xi$ is the acceleration efficiency. The cooling time of electrons can be expressed by

$$
\frac{1}{\tau_{\text {cool }}}=\frac{1}{\tau_{\text {syn }}}+\frac{1}{\tau_{\text {IC }}} .
$$

Then, the cooling time can be expressed by only the synchrotron cooling time

$$
\tau_{\text {cool }}=\tau_{\text {syn }} /(1+\eta)
$$

where $\eta \equiv \tau_{\text {syn }} / \tau_{\mathrm{IC}}, \tau_{\mathrm{syn}}=E_{e}^{\prime} / \dot{P}_{\mathrm{syn}}, \dot{P}_{\mathrm{syn}}=4 \pi \sigma_{\mathrm{T}} c U_{B} \gamma_{e}^{2} / 3, \sigma_{\mathrm{T}}$ is the Thomson cross section, $c$ is the velocity of light, $U_{B}=B^{2} / 8 \pi$

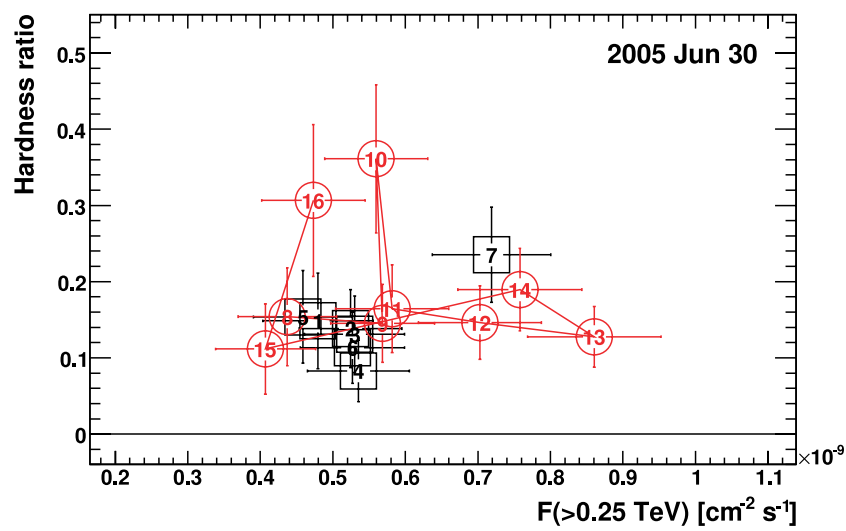

is the energy density of the magnetic field, $\gamma_{e}=E_{e}^{\prime} / m c^{2}$, and $m$ is the electron rest mass. The parameter $\eta$ corresponds to the ratio of the power emitted by electrons in IC and synchrotron processes, respectively (which is reciprocal to the corresponding cooling times $\left.\tau_{\text {syn }} / \tau_{\text {IC }}\right)$. The MAGIC $\gamma$-ray and corresponding $R X T E$ ASM X-ray data permit us to constrain $\eta$ to $\lesssim 0.7$. The modeling of the SED presented in $\S 5.5$ (see Fig. 21 below), however, suggests that $\eta$ is more likely of the order of $\sim 0.2$. We therefore used $\eta=0.2$ in all of the following estimates. By comparing equations (9), (10), and (12) and setting $E_{e}^{\prime} \approx E_{\mathrm{TeV}} / \delta \mathrm{TeV}$ (where $\delta$ is the Doppler factor of the relativistically moving emitting plasma blob and $E_{\mathrm{TeV}}$ is the electron energy [in TeV] in the observer's frame), we obtain the condition on the acceleration efficiency of electrons,

$$
\xi \approx 10^{-3} B E_{\mathrm{TeV}}^{2}(1+\eta) \delta^{-2}
$$

If the observed decay timescale of the flare, $\tau_{f}$, is also due to radiative processes, then

$$
\tau_{\text {cool }}=\tau_{f} \delta
$$

Inserting equation (12) into equation (14) and expanding $\tau_{\text {syn }}$ and $\dot{P}_{\text {syn }}$ permits us to estimate $B$ in the cooling region:

$$
B \approx 11.2\left[(1+\eta)\left(E_{\mathrm{TeV}} \tau_{f}\right)\right]^{-1 / 2} \mathrm{G}
$$

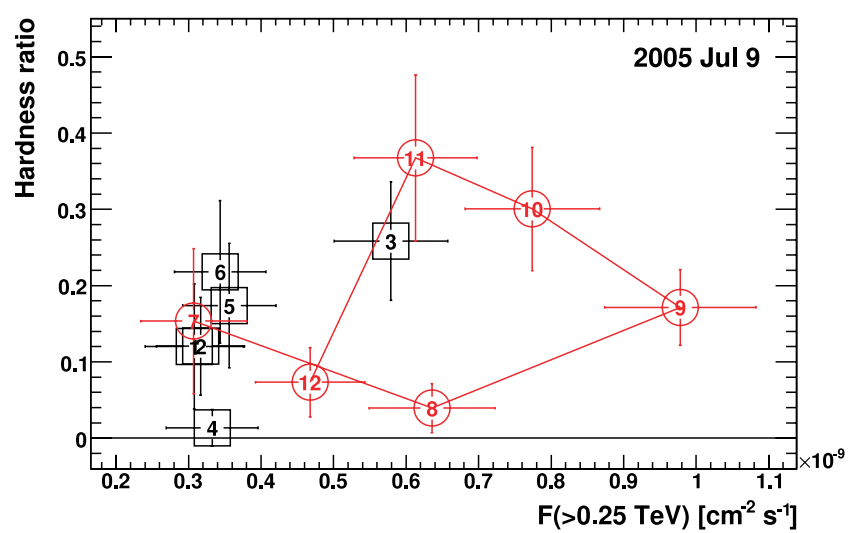

Fig. 17.- Hardness ratio $F(1.2-10 \mathrm{TeV}) / F(0.25-1.2 \mathrm{TeV})$ vs. $F(>0.25 \mathrm{TeV})$ for the nights of June 30 and July 9 . Horizontal and vertical bars denote $1 \sigma$ statistical uncertainties. Black open squares and red open circles denote preburst (stable) and in-burst (variable) emission, respectively (see $\S 3.3$ and Fig. 5). The numbers inside the markers denote the position of the points in the LCs. The consecutive (in time) points of the in-burst LC are connected by red lines for better clarity. 

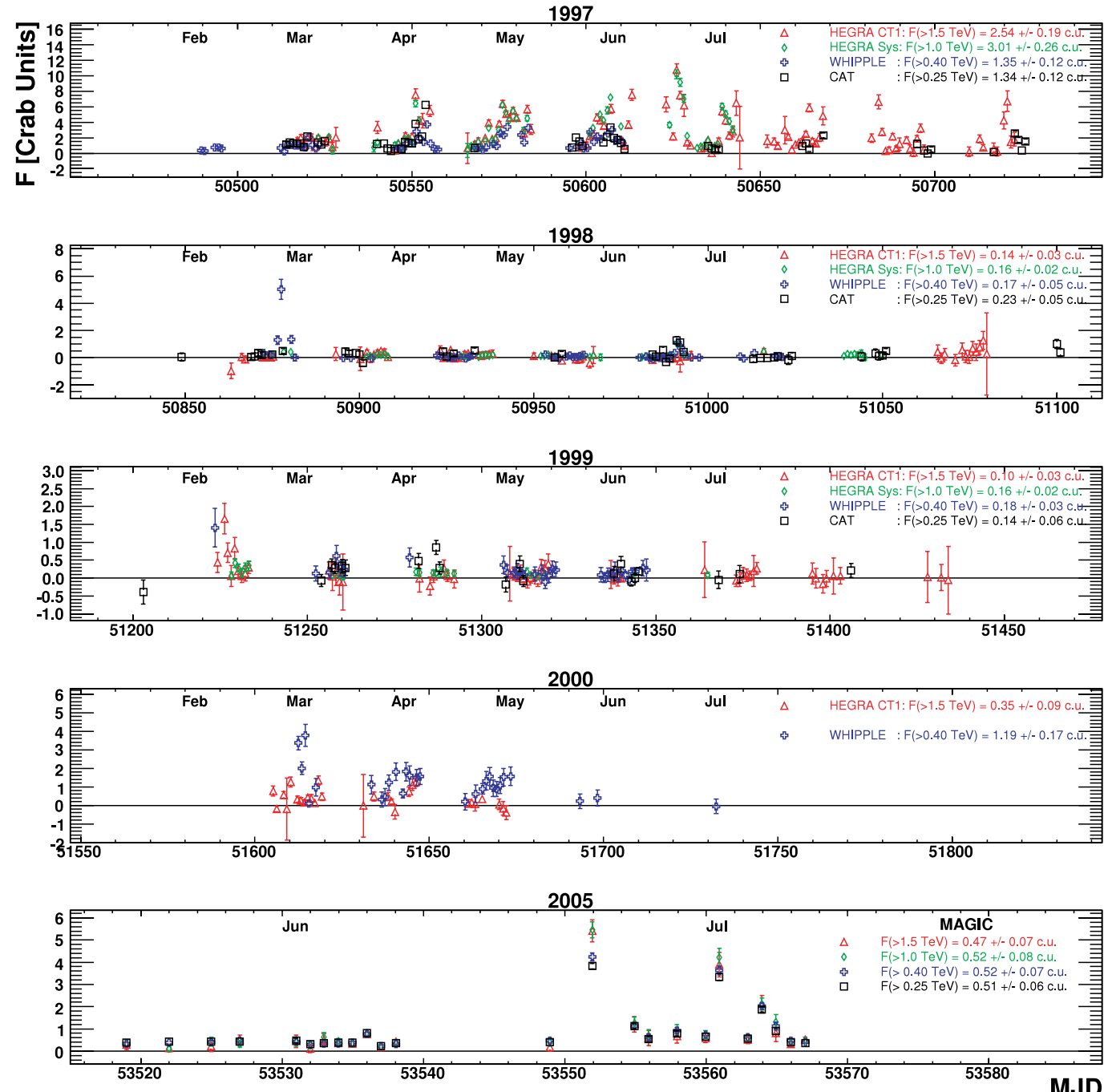

FIG. 18. - Single-night VHE LCs of Mrk 501 obtained with various IACTs during several years. Vertical error bars denote $1 \sigma$ statistical uncertainties. Instruments and corresponding mean fluxes are reported for each observational campaign separately. The MAGIC data were reprocessed to match the energy ranges covered by previous instruments.

with $\tau_{f}$ in seconds. If electron acceleration and cooling are cospatial, equation (13) becomes

$$
\xi \approx 1.1 \times 10^{-2} \frac{E_{\mathrm{TeV}}^{3 / 2}(1+\eta)^{1 / 2}}{\tau_{f}^{1 / 2} \delta^{2}} .
$$

For the parameters of the July 9 flare Mrk 501, the characteristic flux variability time $\tau_{f} \approx 3$ minutes (see $\S 3.3$ ) and $E_{\mathrm{TeV}} \approx 1$, we obtain $B \approx 0.8 \mathrm{G}$ and $\xi \approx 0.9 \times 10^{-3} \delta^{-2}$. Note that this estimate of the magnetic field in the emission (acceleration) region of Mrk 501 is consistent with previous estimates, based on a homogeneous SSC model and the assumption of a very short variability timescale such as for the 1997 April 15-16 flare (see, e.g., Fig. $3 c$ in Bednarek \& Protheroe 1999).

2. Energy-dependent time delay in peak flare emission.-The time delay between the peaks of $F(<0.25 \mathrm{TeV})$ and $F(>1.2 \mathrm{TeV})$ during the July 9 flare can be interpreted as due to the gradual acceleration of electrons in the relativistic blob. As reported in $\S 3.3$, under the assumption that the shape of the flares is the same in the two energy ranges, the time delay is $\Delta \tau_{\mathrm{H}-\mathrm{L}}=4 \pm 1$ minutes.
Within the above framework, the time delay should correspond to the difference between the acceleration times of electrons to energies $E_{\mathrm{TeV}}^{\mathrm{L}} \approx 0.25$ and $E_{\mathrm{TeV}}^{\mathrm{H}} \approx 1.2$, according to

$$
\tau_{\mathrm{acc}}^{\mathrm{H}}-\tau_{\mathrm{acc}}^{\mathrm{L}}=\Delta \tau_{\mathrm{H}-\mathrm{L}} \delta .
$$

Assuming for simplicity that the electron energies are determined by the energies of the emitted VHE radiation, i.e., $E_{e}^{\prime \mathrm{L}} \approx E_{\mathrm{TeV}}^{\prime \mathrm{L}} / \delta$ and $E_{e}^{\prime \mathrm{H}} \approx E_{\mathrm{TeV}}^{\prime \mathrm{H}} / \delta$, we can use equation (10) to model the acceleration time of electrons in equation (17). Finally, by reversing equation (17), we get another limit on the electron acceleration efficiency,

$$
\xi \approx 0.1 \frac{\left(E_{\mathrm{TeV}}^{\mathrm{H}}-E_{\mathrm{TeV}}^{\mathrm{L}}\right)}{B \delta^{2} \Delta \tau_{\mathrm{H}-\mathrm{L}}} .
$$

Applying the observed values of $E_{\mathrm{TeV}}^{\mathrm{H}}, E_{\mathrm{TeV}}^{\mathrm{L}}, \Delta \tau_{\mathrm{H}-\mathrm{L}}$, and the estimate of $B$ from equation (15) (which is valid if electron acceleration and cooling are cospatial), we obtain $\xi \approx 0.5 \times 10^{-3} \delta^{-2}$, which is roughly the same value as determined above using the exponential flux decay time. Note that diffusion and/or spatial 
dishomogeneities might increase the volume where electrons cool down, thus making the magnetic field in the cooling region lower than that of the acceleration region. That would imply that the estimated values for the parameter $\xi$ using equations (16) and (18) are upper and lower limits, respectively.

We conclude that the time delay of the flare peak emission in different ranges of energy can result from the gradual acceleration of the emitting electrons in the blob. The inferred blazar Doppler factors, $\delta \sim 10-15$ (e.g., Costamante \& Ghisellini 2002), imply a relatively inefficient acceleration, $\xi \sim 10^{-5}$. This value is significantly lower than required by the observations of $\gamma$-ray emission from the pulsar wind nebulae in which leptons are accelerated in the shock wave in the relativistic pulsar wind. For example, in the Crab Nebula $\xi$ has to be of the order of $\sim 0.1$, since leptons are accelerated clearly above $10^{3} \mathrm{TeV}$ (approximately $10 \%$ of the maximum available potential drop through the pulsar magnetosphere). Therefore, the physics of the acceleration process in the relativistic jets of BL Lac objects and relativistic shocks in the pulsar wind nebulae may differ significantly.

A somewhat more speculative issue that blazar emission permits us to explore concerns nonconventional physics. Energydependent arrival times are predicted by several models of quantum gravity, which quantify the first-order effects of the violation of Lorentz symmetry. One could, therefore, speculate that the observed time difference is explained by such models, although source-inherent effects could certainly not be excluded. A more detailed investigation of such interpretations of our data is still going on.

\subsection{Interpretation of the Spectral Shape Variations}

The observed correlation between spectral shape and (bolometric) luminosity is naturally accounted for in the SSC scenario. Pian et al. (1998) and Tavecchio et al. (2001) discuss the SED variations of Mrk 501 during the giant 1997 flare. During the 1997 flare, the $0.1-200 \mathrm{keV}$ band synchrotron spectrum became exceptionally flat ( photon spectral index $a<1$ ), peaking at $\gtrsim 100 \mathrm{keV}$, a shift to higher frequencies by a factor of 100 from previous, more quiescent states. The VHE data (from the Whipple, HEGRA, and CAT telescopes) showed a progressive hardening from the baseline state $(a>2)$ through a more active state to a flaring state $(a \sim 2)$. In the SSC scenario, these flux-dependent spectral changes implied that a drastic change in the electron spectrum caused the increase in emitted power: a freshly injected electron population has a flatter high-energy slope and a higher maximum energy than an aging population, which causes a shift of the SED to higher frequencies.

In $\S 4.4$ we reported that the spectrum of Mrk 501 hardens not only on long timescales, with the overall emitted flux, but also during the shorter events of June 30 and July 9. The burst from July 9 showed a remarkable variability, and the evolution of the hardness ratio with the flux (Fig. 17, right panel) contains valuable information about the dynamics of the source. In the preburst phase, the hardness ratio does not vary significantly; yet during the burst phase, it varies following a clear loop pattern rotating counterclockwise. As pointed out by Kirk \& Mastichiadis (1999), one expects to have this behavior for a flare where the variability, acceleration, and cooling timescales are similar, which implies that, during this flare, the dynamics of the system is dominated by the acceleration processes, rather than by the cooling processes. Consequently, the emission propagates from lower to higher energy, so the lower energy photons lead the higher energy ones (that is the so-called hard lag). This indeed agrees well with the argumentation given in $\S 5.2$, where the time delay between $E_{>1.2} \mathrm{TeV}$ and $E_{<0.25 \mathrm{TeV}}$ is shown to be consistent with the gradual acceleration of the electrons.

In a systematic study performed by Gliozzi et al. (2006) using X-ray data from 1998 to 2004, this behavior was not observed on more typical (longer) flares, where actually the opposite behavior (clockwise rotation) was indicated. This might point to the fact that these physical processes might be responsible only for the shortest flux variations, and not for the variability on longer timescales.

\subsection{Interpretation of the Increased Variability with Energy}

In the SSC framework, the variability observed in the VHE emission brings information about the dynamics of the underlying population of relativistic electrons (and possibly positrons). In this context, the general variability trend reported in $\S 3.4$ is interpreted by the fact that the VHE $\gamma$-rays (as well as the $\mathrm{X}$-rays) are produced by more energetic particles, which are characterized by shorter cooling timescales, causing the higher variability amplitude observed at the highest energies. It is worth noticing that such an injection of high-energy particles would produce a shift in the IC peak, which is indeed observed during this observing campaign, as reported in $\S \S 4$ and 5.5.

\subsection{Spectra Corrected for the EBL Absorption}

In this section we correct the measured spectra for the EBL absorption. To this purpose we use Kneiske et al. (2004; "Low"). A correction using the EBL models of Aharonian et al. (2006a) and Primack et al. (2005) gave very similar results, while using Kneiske et al. (2004; "Best") and Stecker et al. (2006, 2007) gave slightly larger energy fluxes above $1 \mathrm{TeV} .^{43}$ Figure 19 shows the spectra from the active nights June 30 and July 9 before/after correction for the EBL absorption. In spite of our proximity to Mrk 501, the effect of the EBL is not negligible, and the spectral peak moves to higher energies.

The locations of the spectral peaks (calculated using eqs. [7] and [8]) are shown as a function of $F(>0.15 \mathrm{TeV})$ in Figure 20 for the flaring nights before and after the EBL correction. The figure seems to indicate a displacement of the peak location with the increasing flux, yet the error bars are too large to be conclusive. On the other hand, the peak location is certainly at $<0.1 \mathrm{TeV}$ when Mrk 501 is in a low state (see $\S 4.2$ ). Hence, there is evidence for an overall peak location versus luminosity trend.

Figure 21 shows the June 30 and July 9 spectra, as well as the mean high-, medium-, and low-flux spectra (see $\S 4.2$ ), EBL deabsorbed using the Low model of Kneiske et al. (2004). The $\mathrm{X}$-ray fluxes measured by RXTE ASM and the optical flux observed by the KVA Telescope are also shown. The optical flux from the host galaxy, estimated by Nilsson et al. (2007) to be $12.0 \pm 1.2 \mathrm{mJy}$, has been subtracted.

The best-fitting one-zone, homogeneous SSC models of Mrk 501 's intrinsic spectrum for the highest state of the source (corresponding to the active night of June 30) and for the lowest state (the mean spectrum corresponding to the low-flux bin; see Fig. 13) are displayed in Figure 21. The fit parameters (electron population's break and $\mathrm{max} / \mathrm{min}$ energies, high/low- $E$ spectral slopes, normalization: $E_{\mathrm{br}}, E_{\max }, E_{\min }, n_{1}, n_{2}, K$; plasma blob's radius, magnetic field, Doppler factor: $R, B, \delta$ ) are reported in Table 9 (for details on the model see Tavecchio et al. 2001). It should be noted that two different fits to the high-state spectrum are possible, whose main differing parameters are, respectively, $(\delta, B)=(25,0.23 \mathrm{G})$ (solid line) and $(50,0.053 \mathrm{G})$ (dashed line), which show that fairly different synchrotron peaks are possible within our X-ray and

\footnotetext{
43 The difference increases with the energy, being $\sim 50 \%$ at $5 \mathrm{TeV}$.
} 

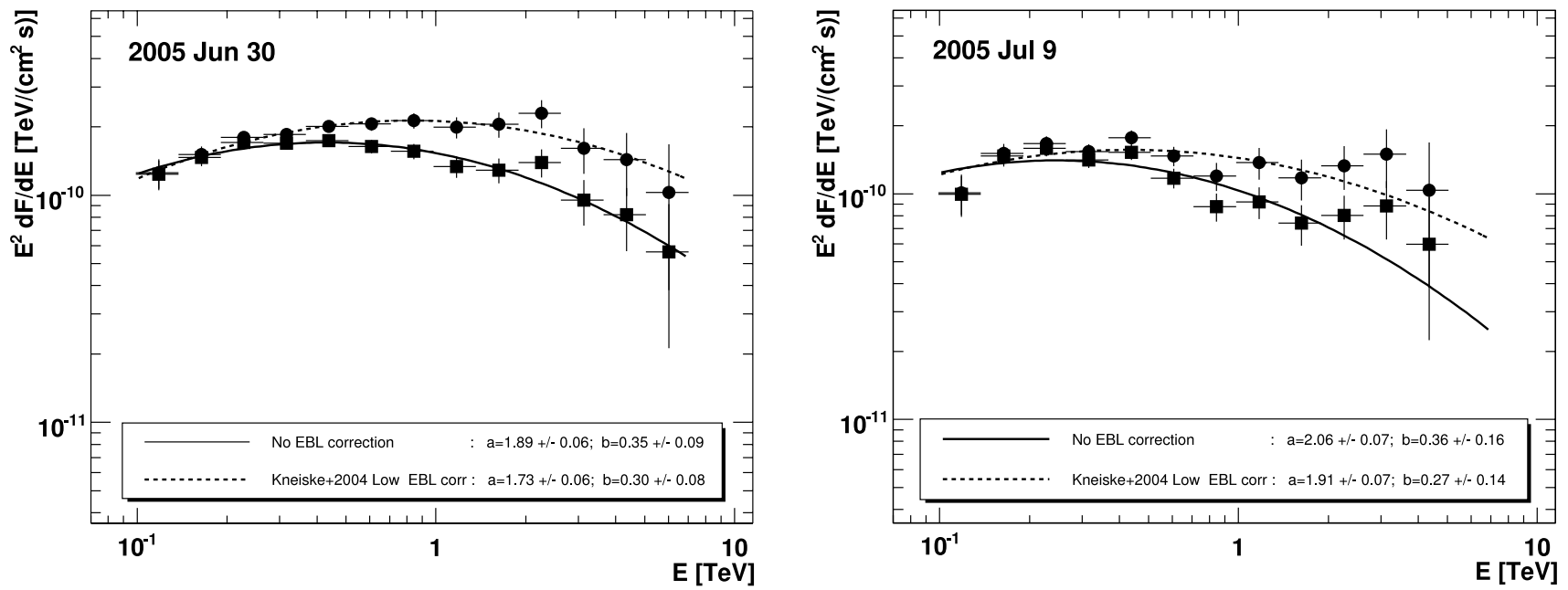

FIG. 19.- Spectra of Mrk 501 in the nights of June 30 (left) and July 9 (right) when the source was flaring. Bars are as in Fig. 13. The spectra have been corrected for EBL absorption using the Kneiske et al. (2004) Low EBL model. The lines show log-parabolic fits (see eq. [6]), whose corresponding parameters are reported in the insets. [See the electronic edition of the Journal for a color version of this figure.]

(EBL corrected) TeV data. Spectrally more extended X-ray data would probably have solved the degeneracy. The optical data, too, do not lift the degeneracy: once the optical light contribution of the underlying host galaxy is subtracted, the observed energy flux is rather compatible with the SED models for the different activity states. ${ }^{44}$ The fit to the low-state spectrum is characterized, perhaps unsurprisingly, by a change of the internal physical conditions of the emitting plasma blob rather than by a change of its bulk attributes (blob size and relativistic Doppler factor): the low state is characterized, with respect to the high state, by lower $\max /$ break energies and normalization of the electron population, as well as by a somewhat stronger magnetic field. One nice consistency feature of all the fits is that, in all cases, the radius of the plasma blob, $R=10^{15} \mathrm{~cm}$, implies a crossing time $t_{\mathrm{cr}}=R / \delta c$,

\footnotetext{
44 The measured optical flux might have contributions from regions outside the one producing the radiation at X-ray and $\gamma$-ray frequencies.
}

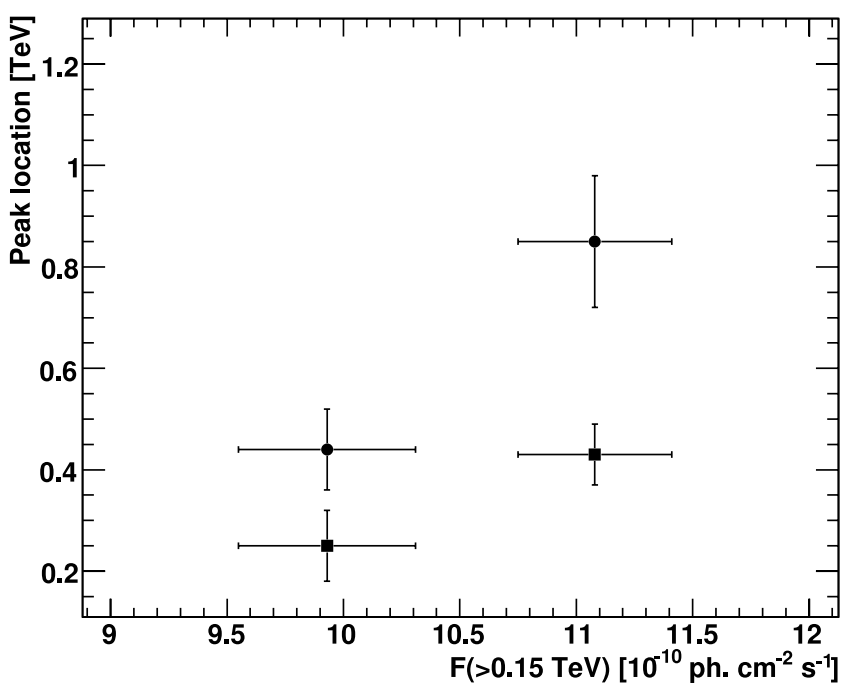

Fig. 20.-Spectral peak location vs. flux above $0.15 \mathrm{TeV}$ for the two flaring nights (June 30 and July 9). The spectra were fitted with eq. (6) (see Fig. 19), and the peak location and its associated error were calculated using eqs. (7) and (8). The squares correspond to the observed spectra, and the circles correspond to the spectra after correction for the EBL absorption using the Kneiske et al. (2004) Low EBL model. [See the electronic edition of the Journal for a color version of this figure.] comparable to that inferred from the observed duration ( $20 \mathrm{~min}-$ utes) of the flare, $\Delta t_{\text {flare }}$.

The SED models for Mrk 501 derived and discussed in this section can be compared with some previous published models, like Pian et al. (1998), with $(\delta, B) \simeq(15,0.8 \mathrm{G})$ from one-zone SSC modeling of 1997 SEDs, with flatter/steeper electron distributions for active/quiescent phases; Tavecchio et al. (2001), with $(\delta, B) \simeq(8-20,0.5-1 \mathrm{G})$ from one-zone SSC modeling of historical quiescent SED and $(\delta, B) \simeq(7,1 \mathrm{G})$ (and very high break

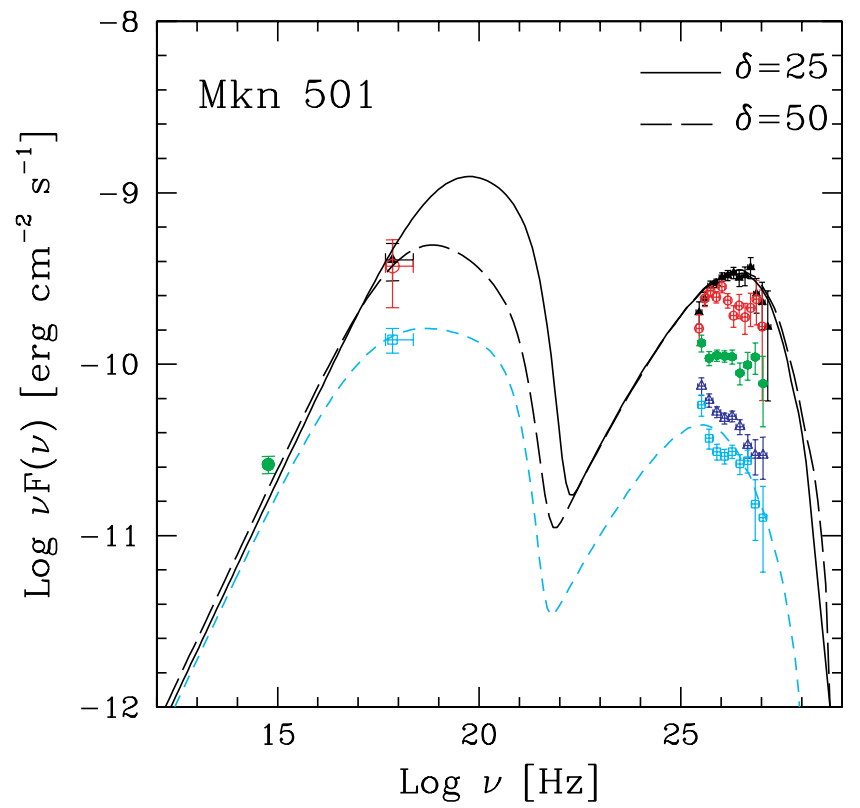

FIG. 21.-Overall SED from Mrk 501. The optical data from the KVA Telescope are represented with a green filled circle; the X-ray data from RXTE ASM are depicted with a black filled triangle for June 30 , red open circle for July 9 , and light blue open square for the other nights (combined); the VHE data from MAGIC are represented as black filled triangles (June 30), red open circles (July 9), green filled circles ("high-flux" data set), dark blue open triangles ("medium-flux" data set), and light blue open squares ("low-flux" data set). See $\S 4.2$ for definitions of high-, medium-, and low-flux data sets. Vertical error bars denote $1 \sigma$ statistical uncertainties. The VHE spectra are corrected for EBL extinction using the Kneiske et al. (2004) Low EBL model. The highest and the lowest states were fitted with a one-zone SSC model (described in Tavecchio et al. 2001). See Table 9 and $\S 5.5$ for details of the modeling. 
TABLE 9

SSC Model Parameters

\begin{tabular}{|c|c|c|c|c|c|c|c|c|c|}
\hline Spectrum & $\gamma_{\min }$ & $\gamma_{\mathrm{br}}$ & $\gamma_{\max }$ & $\mathrm{n} 1$ & $\mathrm{n} 2$ & $\begin{array}{c}B \\
(\mathrm{G})\end{array}$ & $\begin{array}{c}K \\
\left(\text { particles } \mathrm{cm}^{-3}\right)\end{array}$ & $\begin{array}{c}R \\
(\mathrm{~cm})\end{array}$ & Doppler Factor \\
\hline Jun 30 & 1 & $10^{6}$ & $10^{7}$ & 2 & 3.5 & 0.23 & $7.5 \times 10^{4}$ & $10^{15}$ & 25 \\
\hline 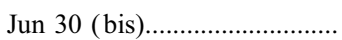 & 1 & $5 \times 10^{5}$ & $10^{7}$ & 2 & 3.5 & 0.053 & $7.0 \times 10^{4}$ & $10^{15}$ & 50 \\
\hline 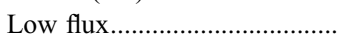 & 1 & $10^{5}$ & $5 \times 10^{6}$ & 2 & 3.2 & 0.31 & $4.3 \times 10^{4}$ & $10^{15}$ & 25 \\
\hline
\end{tabular}

energy) for the active SED; Bednarek \& Protheroe (1999), with $(\delta, B) \simeq(12-36,0.07-0.6 \mathrm{G})$ from 1997 SEDs modeled with one-zone SSC requiring $\gamma$-ray transparency of the emitting blob; Kataoka et al. (1999), with $(\delta, B) \simeq(15,0.2 \mathrm{G})$ from one-zone SSC modeling of simultaneous 1996 SED; and Katarzyński et al. (2001), with $(\delta, B)=(14,0.2 \mathrm{G})$ from SSC modeling of nonsimultaneous broadband SED. The SED of Mrk 501 can also be modeled by less conventional approaches, requiring magnetic fields in the emission region smaller than $0.005 \mathrm{G}$ (Krawczynski 2007). We should, however, remember that for this highly variable source, constraints derived for some epochs may not apply to other epochs (the simple one-zone model has nine free parameters!): in particular, most published models refer to the giant 1997 flare; hence, comparisons with our results may not be straightforward. However, Tavecchio et al. (2001) modeled different emission states of Mrk 501 in 1997, 1998, and 1999 by just changing the electron energy distribution (slopes, break energy, number density) and keeping the other parameters frozen, similarly to what was done here (see in Table 9 the two states represented by solid lines in Fig. 21). It is also worth mentioning the work done by Krawczynski et al. (2002), in which the energy spectra (for different days) were modeled using a time-dependent code.

It should be remarked that in $\mathrm{TeV}$ blazars, while the bright and rapidly variable VHE emission implies that at the scales where this emission originates ( $\sim 0.1 \mathrm{pc}$ from the jet apex) the jet is highly relativistic $(\delta \sim 10-20$ with no EBL absorption correction, and $\delta \lesssim 50$ with correction), at VLBI $(\sim 1 \mathrm{pc})$ scales the jets are relatively slow (see Ghisellini et al. 2005 and references therein). Hence, to reconcile the high $\delta$-values derived from VHE data with the much lower $\delta$-values derived from VLBI radio measurements, the jets of $\mathrm{TeV}$ blazars must either undergo severe deceleration (Georganopoulos \& Kazanas 2003) or be structured radially as a twovelocity (inner spine plus outer layer) flow (Ghisellini et al. 2005).

\section{CONCLUDING REMARKS}

In this work we have undertaken a systematic study of the temporal and spectral variability of the nearby blazar Mrk 501 with the MAGIC Telescope at energies $>0.1 \mathrm{TeV}$. During 24 observing nights between 2005 May and July, all of which yielded significant detections, we measured fluxes and spectra at levels of baseline activity ranging from $<0.5$ to $>1 \mathrm{crab}$. During two nights, on June 30 and July 9 , Mrk 501 underwent a clearly active state with a $\gamma$-ray emission $>3$ crab and flux-doubling times of $\sim 2$ minutes. The $\sim 20$ minute long flare of July 9 showed an indication of a $4 \pm 1$ minute time delay between the peaks of $F(<0.25 \mathrm{TeV})$ and $F(>1.2 \mathrm{TeV})$, which may indicate a progressive acceleration of electrons in the emitting plasma blob. An overall trend of harder spectra for higher flux is clearly seen on intranight, night-by-night, and longer term timescales. The VHE $\gamma$-ray variability was found to increase with energy, regardless of whether the source is in active or quiescent state, and it is significantly higher than the variability at $\mathrm{X}$-ray frequencies. A spectral peak, at a location dependent on source luminosity, was clearly observed during the active states. All these features are naturally expected in SSC models of blazar VHE emission. There are no simultaneous good-quality X-ray measurements during the MAGIC observations. As a consequence, the SSC model of the X-ray/VHE SED of Mrk 501 in an active state is not unequivocally constrained, but it still restricts the emitting plasma blob to have Doppler factors in the range of 25-50 and magnetic fields in the range of $0.05-0.5 \mathrm{G}$.

We would like to thank the IAC for the excellent working conditions on the La Palma Observatory Roque de los Muchachos. We are grateful to the RXTE ASM team for their quick-look results. The support of the German BMBF and MPG, the Italian INFN, and the Spanish CICYT is gratefully acknowledged. This work was also supported by ETH research grant TH-34/04-3 and by Polish grant MNiI 1P03D01028. In addition, the authors want to thank Deirdre Horan, Martin Tluczykont, and Frédéric Piron for providing Mrk 501 data from WHIPPLE, HEGRA CT1, and HEGRA CT System and CAT, respectively, and for useful discussions.
Aharonian, F. A. 2000, NewA, 5, 377

Aharonian, F. A., et al. 1999a, A\&A, 342, 69

. 1999b, A\&A, 349, 11

2001, ApJ, 546, 898

2003, A\&A, 403, L1

2004, ApJ, 614, 897

2006a, Nature, 440, 1018

2006, Science, 314, 1424

2007, ApJ, 664, L71

Albert, J. 2006, preprint (astro-ph/0612385)

Anykeyev, V. B., et al. 1991, Nucl. Instrum. Methods Phys. Res. A, 303, 350

Atoyan, A. M., \& Dermer, C. D. 2003, ApJ, 586, 79

Baixeras, C., et al. 2004, Nucl. Instrum. Methods Phys. Res. A, 518, 188

Bassani, L., \& Dean, J. 1981, Nature, 294, 332

Beall, J. H., \& Bednarek, W. 1999, ApJ, 510, 188

Bednarek, W. 1993, ApJ, 402, L29

Bednarek, W., \& Protheroe, R. J. 1997a, MNRAS, 287, L9

. 1997b, MNRAS, 290, 139

\section{REFERENCES}

Bednarek, W., \& Protheroe, R. J. 1999, MNRAS, 310, 577

Bertero, M. 1989, Advances in Electronics and Electron Physics, Vol. 75 (New York: Academic)

Bityukov, S., et al. 2006, On the Combining Significances (arXiv: physics/0612178) Blandford, R. D., \& Königl, A. 1979, ApJ, 232, 34

Blandford, R. D., \& Rees, M. J. 1978, in Proc. Pittsburgh Conference on BL Lac Objects, ed. A. Wolfe (Pittsburgh: Univ. Pittsburgh Press), 328

Böttcher, M. 2002, in Proc. XXII Moriond Astrophysics Meeting "The Gamma-ray Universe," ed. A. Goldwurm, D. Neumann, \& J. Tran Thanh Van (Hanoi: Gioi), 151

Bradbury, S. M., et al. 1997, A\&A, 320, L5

Cortina, J., et al. 2005, in Proc. 29th Int. Cosmic Ray Conf. (India), 359

Costamante, L., \& Ghisellini, G. 2002, A\&A, 384, 56

Dar, A., \& Laor, A. 1997, ApJ, 478, L5

Dermer, C. D., \& Schlickeiser, R. 1993, ApJ, 416, 458

Djanati-Ataï, A., et al. 1999, A\&A, 350, 17

Dondi, L., \& Ghisellini, G. 1995, MNRAS, 273, 583

Edelson, R., et al. 2002, ApJ, 568, 610 
Gaidos, J. A., et al. 1996, Nature, 383, 319

Gaug, M. 2006, Ph.D. thesis, IFAE Barcelona

Georganopoulos, M., \& Kazanas, D. 2003, ApJ, 594, L27

Ghisellini, G., \& Madau, P. 1996, MNRAS, 280, 67

Ghisellini, G., Tavecchio, F., \& Chiaberge, M. 2005, A\&A, 432, 401

Gliozzi, M., et al. 2006, ApJ, 646, 61

Hayashida, N., et al. 1998, ApJ, 504, L71

Hillas, A. M. 1985, in Proc. 19th Int. Cosmic Ray Conf. (La Jolla), 445

Hillas, A. M., et al. 1998, ApJ, 503, 744

Hrupec, D. 2007, Ph.D. thesis, Univ. Zagreb

Kataoka, J., et al. 1999, ApJ, 514, 138

Katarzyński, K., Sol, H., \& Kus, A. 2001, A\&A, 367, 809

Kirk, J. G., \& Mastichiadis, A. 1999, Astropart. Phys., 11, 45

Kneiske, T. M., et al. 2004, A\&A, 413, 807

Kranich, D. 2000, Ph.D. thesis, MPI Munich

Krawczynski, H. 2007, ApJ, 659, 1063

Krawczynski, H., Coppi, P. S., \& Aharonian, F. 2002, MNRAS, 336, 721

Krawczynski, K., et al. 2004, ApJ, 601, 151

Li, T., \& Ma, Y. 1983, ApJ, 272, 317

Mannheim, K. 1993, A\&A, 269, 67

Mannheim, K., \& Biermann, P. L. 1992, A\&A, 253, L21

Maraschi, L., Ghisellini, G., \& Celotti, A. 1992, ApJ, 397, L5

Marscher, A. P., \& Gear, W. K. 1985, ApJ, 298, 114

Massaro, E., et al. 2004, A\&A, 413, 489 . 2006, A\&A, 448, 861

Mattox, J. R., et al. 1993, ApJ, 410, 609

McBreen, B. 1979, A\&A, 71, L19

Mücke, A., et al. 2003, Astropart. Phys., 18, 593

Multi-Messenger Group 2006, Gamma-Ray Data

Nandra, K., et al. 1997, ApJ, 476, 70

Nilsson, K., et al. 2007, A\&A, in press (arXiv: 0709.2533)

Osone, S. 2006, Astropart. Phys., 26, 209
Paneque, D. 2004, Ph.D. thesis, MPI Munich

Pian, E., et al. 1998, ApJ, 492, L17

Piron, F. 2000, Ph.D. thesis, LPNHE Ecole Polytechnique 2003, in Very High-Energy Phenomena in the Universe, Proc. XXXVI Rencontres de Moriond, ed. M. Boër \& J. Tran Thanh Van (Les Arcs: Thé Giói), 73

Poe, G. L., Giraud, K. L., \& Loomis, J. B. 2005, Am. J. Agricultural Economics, 87 (2), 353

Pohl, M., \& Schlieckeiser, R. 2000, A\&A, 354, 395

Primack, J., et al. 2005, in AIP Conf. Proc. 745, High Energy Gamma-Ray Astronomy, ed. F. A. Aharonian, H. J. Völk, \& D. Horns (New York: AIP), 23

Quinn, J., et al. 1996, ApJ, 456, L83 1999, ApJ, 518, 693

Ravasio, M., et al. 2004, A\&A, 424, 841

Rees, M. J. 1984, ARA\&A, 22, 471

Rieger, F. M., \& Mannheim, K. 2000, A\&A, 359, 948 2003, A\&A, 397, 121

Samuelson, F. W., et al. 1998, ApJ, 501, L17

Schweizer, T. 2004, Ph.D. thesis, IFAE Barcelona

Sikora, M., et al. 1994, ApJ, 421, 153

Stecker, F. W., et al. 2006, ApJ, 648, 774 2007, ApJ, 658, 1392

Tavecchio, F., et al. 2001, ApJ, 554, 725

Taylor, J. R. 1997, An Introduction to Error Analysis (Mill Valley: University Science Books)

Ulrich, M. H., et al. 1997, ARA\&A, 35, 445

Urry, C. M., \& Padovani, P. 1995, PASP, 107, 803

Vaughan, S., et al. 2003, MNRAS, 345, 1271

Wagner, R. M., et al. 2005, in Proc. 29th Int. Cosmic Ray Conf. (Pune), 163

Xue, Y., \& Cui, W. 2005, ApJ, 622, 160

Zhang, Y. H., et al. 2006a, ApJ, 637, 699 . 2006b, ApJ, 651, 782 